\title{
ON THE DEGREE OF POLYNOMIAL APPROXIMATION TO HARMONIC AND ANALYTIC FUNCTIONS
}

\author{
BY \\ J. L. WALSH, W. E. SEWELL, AND H. M. ELLIOTT(1)
}

1. Introduction. In the plane of the complex variable $z=x+i y$, a function $u(z)$ harmonic $\left({ }^{2}\right)$ in the interior of the Jordan curve $C$ and continuous in the corresponding closed region $\bar{C}$ can be represented in $\bar{C}$ by a uniformly convergent series of harmonic polynomials [Walsh 2,3$]\left({ }^{3}\right)$. Let $C_{R}$ denote the image in the $z$-plane of the circle $|w|=R(>1)$ under the conformal map of $|w|>1$ of the $w$-plane onto the exterior of $C$ so that the points at infinity in the two planes correspond to each other. If $u(z)$ is harmonic throughout the interior of $C_{R}$, then there exist [Walsh 1,5$]$ harmonic polynomials $p_{n}(z)$ of respective degrees $n=1,2, \cdots$, such that we have

$$
\limsup _{n \rightarrow \infty}\left[\max \left|u(z)-p_{n}(z)\right| \text { on } C\right]^{1 / n} \leqq 1 / R ;
$$

conversely, if harmonic polynomials $p_{n}(z)$ of respective degrees $n$ exist so that (1.01) is satisfied, the sequence $p_{n}(z)$ converges throughout the interior of $C_{R}$ and the function $u(z)$ if suitably defined exterior to $C$ is consequently harmonic throughout the interior of $C_{R}$. It is the purpose of the present paper to sharpen these results by studying the more delicate problem of relating (i) continuity properties of $u(z)$ (in the form of Lipschitz $\left(^{4}\right)$ conditions on the function or its derivatives) on $C$ or $C_{R}$ to (ii) degree-of convergence in $\bar{C}$ of the sequences $p_{n}(z)$ and $\pi_{n}(z)$, where $\pi_{n}(z)=p_{n}(z)+i q_{n}(z)$ is a polynomial in $z$ of degree $n$.

Presented to the Society April 26, 1940 and October 30, 1948 under a different title; received by the editors November 22, 1948. Abstract published in Proc. Nat. Acad. Sci. U.S.A. vol. 35 (1949) pp. 59-62.

(1) Dr. H. M. Elliott is a Vassie James Hill A.A.U.W. Fellow.

(2) A function $u(z)=u(x, y)$ is harmonic in a region if it is continuous there together with its first and second partial derivatives, and if $\left(\partial^{2} u / \partial x^{2}\right)+\left(\partial^{2} u / \partial y^{2}\right)=0$. A function is harmonic at $a$ point if it is harmonic in some neighborhood of that point. Throughout this paper we shall, without further mention, understand by a "harmonic function" or "harmonic polynomial" a real harmonic function or polynomial; $u(z)$ will always denote a real function.

(3) Numbers in brackets refer to the bibliography at the end of this paper.

(4) A function $F(z)$ is said to satisfy a Lipschitz condition of order $\alpha$ on a set $E$ if for any two points $z_{1}, z_{2}$ of $E$ we have $\left|F\left(z_{1}\right)-F\left(z_{2}\right)\right| \leqq L\left|z_{1}-z_{2}\right|^{\alpha}$, where $L$ is a constant independent of $z_{1}$ and $z_{2}$. Henceforth the letters $L$ and $M$ with or without subscripts will always denote constants which may vary from one inequality to another and which may depend on the point sets involved, but which are always independent of $z$ and of indices $m$ and $n$. The letter $K$ with or without a subscript will similarly denote a constant which may depend on $n$ and the point sets involved but which is independent of $z$. 
We denote by Problem $\alpha$ the study of the relation between continuity properties of $u(z)$ on $C$ and degree of convergence in $\bar{C}$, by Problem $\beta$ the study of the relation between continuity properties of $u(z)$ on $C_{R}$ and degree of convergence in $\bar{C}$, by Problem $\gamma$ the study of the behavior on the curves $C_{R}$ of the special polynomials of Problem $\beta$. To be more explicit as to our results, we show (Problem $\alpha$ ) under suitable restrictions on $C$ that if the $k$ th derivative $\partial^{k} u / \partial s^{k}$ of $u(z)$ with respect to arc-length $s$ satisfies on $C$ a Lipschitz condition of order $\alpha(0<\alpha \leqq 1)$, then a properly chosen sequence $\pi_{n}(z)$ converges in $\bar{C}$ to $f(z)=u(z)+i v(z)$ with degree of convergence $1 / n^{k+\alpha}$; conversely, if a sequence $p_{n}(z)$ converges to $u(z)$ in $\bar{C}$ with this degree of convergence, then $f^{(k)}(z)$ satisfies on $C$ a Lipschitz condition of order $\alpha$ if $\alpha<1$. We show also (Problem $\beta$ ) that if $u(z)$ is continuous on $\bar{C}_{R}$ and if $\partial^{k} u / \partial s^{k}$ satisfies a Lipschitz condition of order $\alpha$ on $C_{R}$, then a suitable sequence $\pi_{n}(z)$ converges to $f(z)$ on $\bar{C}$ with a degree of convergence $1 / n^{k+\alpha} R^{n}$; and conversely if a sequence $p_{n}(z)$ converges to $u(z)$ on $\bar{C}$ with this degree of convergence, then $f^{(k-1)}(z)$ satisfies on $C_{R}$ a Lipschitz condition of order $\alpha$ if $\alpha<1$.

A classification of functions analytic in the unit circle due to Hardy and Littlewood expressed in terms of Lipschitz conditions and asymptotic inequalities was used by Walsh and Sewell [4] as the basis of the study of approximation to analytic functions by polynomials in $z$. This same classification of analytic functions yields by separation into real and pure imaginary parts a corresponding classification of functions harmonic in the unit circle; we use both classifications in the present paper, study their relations, and from a given degree of approximation to a harmonic function $u(z)$ derive results on the class of the corresponding analytic function $f(z)$ and on degree of approximation to $f(z)$.

The entire theory here developed is closely related to and in the main contains the analogous theory for approximation to analytic functions by polynomials in the complex variable, an exposition of which is given by Sewell [1]. Our results for an analytic Jordan curve $C$ are in each case $(0<\alpha \leqq 1)$ actually stronger than those which have previously been established in the analogous theory; for we assume continuity properties merely of $u(z)$ and derive the previous results on degree of approximation to $f(z)$; and conversely we assume various degrees of approximation merely to $u(z)$ by harmonic polynomials and derive the previous results on the continuity properties of $f(z)$. Thus the present theory is by no means merely an obvious application of the former one, although incomplete portions of our results are thus obtainable. Examples of essential difficulties in establishing the new theory from the old one are: (a) if $u(z)$ satisfies a Lipschitz condition of order $\alpha(0<\alpha<1)$ on an analytic Jordan curve $C$, so also does its conjugate $v(z)$; approximation to $u+i v$ by polynomials in $z$ then yields our results on approximation to $f(z)$ by polynomials in $z$, but this method fails to yield our results in the case $\alpha=1$; (b) if a sequence of harmonic polynomials converges uniformly in a 
closed region, the sequence of conjugate harmonic polynomials need not converge there, and if convergent need not have the same degree of convergence.

The theory of approximation to functions of a real variable $\theta$ by trigonometric polynomials has long been highly developed, due primarily to the work of Lebesgue, Jackson, S. Bernstein, de la Vallée Poussin, and Montel, and is essentially equivalent to approximation by harmonic polynomials in the closed interior of the unit circle, so partial results in the latter theory are available at once. The study of approximation to functions of a real variable served as a pattern (primarily as to results rather than method) in the study of approximation to functions of a complex variable $z$ by polynomials in $z$, a theory commenced by Runge, Hilbert, Faber, Montel, Fejér, Szegö, Carleman, and elaborated by Walsh, Russell, Sewell, and Curtiss. The latter theory serves remarkably well as a pattern in the present study, both as to results and method; but as we have already stated, the present work is by no means a mere imitation or repetition of that previous theory.

The older work on approximation to a real periodic function $F(\theta)$ by trigonometric polynomials has recently been complemented by Zygmund [2], who shows that for a continuous function the inequality

$$
\left|\frac{F(\theta+h)+F(\theta-h)-2 F(\theta)}{h}\right| \leqq M
$$

is a necessary and sufficient condition for order of approximation $1 / n$. Throughout the present treatment, however, we emphasize Lipschitz conditions and other moduli of continuity; since (1.02) is not a condition on the modulus of continuity it is to that extent foreign to the present paper. Nevertheless, Zygmund's methods and results are also of importance in the study of approximation to harmonic and analytic functions, and likewise yield (under suitable restrictions) a necessary and sufficient condition for degree of approximation $1 / n$, as will be shown in a later paper.

In $\$ 2$ we define several classes of harmonic functions and discuss directional derivatives. In $\$ 3$ we investigate the relation between Lipschitz conditions on $u(z)$ and on $f(z)$. In Part I ( $\$ 4-6)$ we study Problems $\alpha$ and $\beta$ for the unit circle. In Part II ( $\$ \S 7-13)$ we study Problems $\alpha, \beta$, and $\gamma$ for more general point sets; $\S \S 7,9$ are devoted to the establishment of inequalities on the moduli of polynomials, $\S 8$ to Problem $\alpha, \S \S 10,11,12$ to Problem $\beta, \S 13$ to Problem $\gamma$. Examples to appraise the sharpness of our results are given in $\S 14$.

2. Continuity classes; derivatives. In our study of approximation, it is convenient to define four classes of functions as follows. Let $C$ be a rectifiable Jordan curve. We shall say that the real function $u(z)$ belongs to the class $L(k, \alpha)$, symbolically $u(z) \in L(k, \alpha)$, on $C$ if $u(z)$ is harmonic interior to $C$ and continuous on $\bar{C}$; if the derivative $\partial^{k} u(z) / \partial s^{k}$ exists $\left(^{5}\right)$ on $C$, where $s$ is arc-

(5) In $\S \S 1-11$ the letter $k$ will be reserved to denote a non-negative integer. 
length measured along $C$; and if $\partial^{k} u(z) / \partial s^{k}$ satisfies on $C$ a Lipschitz condition of order $\alpha, 0<\alpha \leqq 1\left(^{6}\right)$. We shall say that $u(z) \in \log (k, \beta)$ on $C$ if $u(z)$ is harmonic interior to $C$, continuous in $\bar{C}$, and $\partial^{k} u(z) / \partial s^{k}$ exists on $C$ and satisfies the condition, where $\left|z_{1}-z_{2}\right|$ is sufficiently small $\left({ }^{7}\right)$,

$$
\left|\frac{\partial^{k} u\left(z_{1}\right)}{\partial s^{k}}-\frac{\partial^{k} u\left(z_{2}\right)}{\partial s^{k}}\right| \leqq L\left|z_{1}-z_{2}\right||\log | z_{1}-z_{2}|| \beta, \quad z_{1}, z_{2} \text { on } C \text {. }
$$

The complex-valued function $f(z)$ is said to belong to the class $L_{A}(k, \alpha)$ on $C$ if $f(z)$ is analytic interior to $C$, continuous on $\bar{C}$, and if the one-dimensional derivative $f^{(k)}(z)$ exists and satisfies a Lipschitz condition of order $\alpha$ on $C$. Finally, $f(z) \in \log _{A}(k, \beta)$ on $C$ if $f(z)$ is analytic interior to $C$, continuous on $\bar{C}$, and if $f^{(k)}(z)$ exists on $C$ in the one-dimensional sense and satisfies on $C$ a condition of the form (2.01).

If $C$ consists of a finite number of rectifiable Jordan curves, we say $u(z) \in L(k, \alpha)$ on $C$ if $u(z) \in L(k, \alpha)$ on each component of $C$; we make an analogous definition for the classes $L_{A}(k, \alpha), \log (k, \beta)$, and $\log _{A}(k, \beta)$. Furthermore, $f(z) \in L_{A}(k, \alpha)$ on $\bar{C}$ if $f(z) \in L_{A}(k, \alpha)$ on $C$ and $f^{(k)}(z)$, defined on $C$ in the one-dimensional sense and interior to $C$ in the usual way, satisfies a Lipschitz condition of order $\alpha$ on $\bar{C}$. A corresponding definition applies to the class $\log _{A}(k, \beta)$. When we refer to a function $u(z)$ harmonic in several mutually disjoint regions, we do not imply that $u(z)$ is a monogenic harmonic function. A similar remark applies to an analytic function $f(z)$.

We turn now to the question of derivatives. Let $C$ be a Jordan curve; let $f(z)$ be analytic interior to $C$ and continuous on $\bar{C}$. At a point $z_{0}$ interior to $C$ the derivative $f^{\prime}\left(z_{0}\right)$ is uniquely defined due to the analyticity of $f(z)$ at $z_{0}$. If $z_{0}$ is a point on $C$, there are three different limits which may appropriately be considered in studying $f^{\prime}\left(z_{0}\right)$. The one-dimensional derivative of $f(z)$ on $C$ is

$$
\lim _{z \rightarrow z_{0}, z \text { on } C} \frac{f(z)-f\left(z_{0}\right)}{z-z_{0}},
$$

provided this limit exists; this definition is valid for a function defined merely on an arc. The two-dimensional derivative is

$$
\lim _{z \rightarrow z_{0}, z \text { on } \bar{C}} \frac{f(z)-f\left(z_{0}\right)}{z-z_{0}},
$$

provided this limit exists. Finally

${ }^{(6)}$ Clearly the requirement that $u(z)$ satisfy a Lipschitz condition of order $\alpha$ on the unit circle is equivalent to the requirement that $u\left(e^{i \theta}\right)=U(\theta)$ satisfy a Lipschitz condition of order $\alpha$ in $\theta$. The superscript $k$ indicates generically differentiation $k$ times; in particular, the superscript $k=0$ does not alter the function.

(7) The restriction on $\left|z_{1}-z_{2}\right|$ is clearly essential since if $\left|z_{1}-z_{2}\right|=1$, the right side of inequality (2.01) is zero. Henceforth, whenever such a restriction on $\left|z_{1}-z_{2}\right|$ is necessary, it will be assumed without explicit mention. 


$$
\lim _{z \rightarrow z_{0, z} \text { on } \bar{C}} f^{\prime}(z)
$$

may be considered provided, of course, $f^{\prime}(z)$ exists; here $f^{\prime}(z)$ for $z$ on $C$ may be defined by either (2.02) or (2.03).

Definition 2.1. Let $C$ be a rectifiable Jordan curve. We say that the arc and chord of $C$ are infinitesimals of the same order if there exists a number $A>1$ such that if $r$ denotes the distance between any two points $z_{1}, z_{2}$ of $C$ and $s$ denotes the length of the shorter arc (or the common value if the two arcs have the same length) of $C$ joining $z_{1}$ and $z_{2}$, then $s / r \leqq A$.

THEOREM 2.1. Let $C$ be a rectifiable Jordan curve whose arc and chord are infinitesimals of the same order. Let the complex-valued function $f(z)$ be continuous on $C$ and have a continuous derivative on $C$ in the one-dimensional sense with $\left|f^{\prime}(z)\right| \leqq M$. Then for $z_{1}$ and $z_{2}$ on $C$ we have( $\left.{ }^{8}\right)$

$$
\left|\frac{f\left(z_{1}\right)-f\left(z_{2}\right)}{z_{1}-z_{2}}\right| \leqq M A,
$$

where $A$ is the constant of Definition 2.1.

The proof depends on the validity of the equation

$$
f\left(z_{2}\right)-f\left(z_{1}\right)=\int_{z_{1}}^{z_{2}} f^{\prime}(z) d z
$$

where the integral is taken along $C$ and $f^{\prime}(z)$ is the one-dimensional derivative of $f(z)$ on $C$. Let $z_{0}$ be a point on $C$. Set $F(z)=\int_{z_{0}}^{z} f^{\prime}(z) d z$, the integral being taken along $C$. Then for $z, z_{1}$ on $C$

$$
F(z)-F\left(z_{1}\right)-\left(z-z_{1}\right) f^{\prime}\left(z_{1}\right)=\int_{z_{1}}^{z}\left[f^{\prime}(t)-f^{\prime}\left(z_{1}\right)\right] d t .
$$

Since $f^{\prime}(z)$ is continuous on $C$, given an arbitrary $\epsilon>0$, there exists an $\eta>0$ such that for $z, z_{1}$ on $C$ and $\left|z-z_{1}\right| \leqq \eta$, we have $\left|f^{\prime}(z)-f^{\prime}\left(z_{1}\right)\right| \leqq \epsilon / A$. Thus for $\left|z-z_{1}\right| \leqq \eta$

$$
\left|F(z)-F\left(z_{1}\right)-\left(z-z_{1}\right) f^{\prime}\left(z_{1}\right)\right| \leqq(\epsilon / A) \operatorname{arc}\left(z \quad z_{1}\right) \leqq \epsilon\left|z-z_{1}\right| \cdot
$$

It follows that $F^{\prime}(z)$ exists and is equal to $f^{\prime}(z), z$ on $C$, where $F^{\prime}(z)$ and $f^{\prime}(z)$ are one-dimensional derivatives taken along $C$. But if a complex-valued function $g(z)$ has on $C$ a one-dimensional derivative $g^{\prime}(z) \equiv 0$, then $g(z)$ is a constant, for if $z, z+\Delta z$ are on $C$

$$
\lim _{\Delta s \rightarrow 0} \frac{g(z+\Delta z)-g(z)}{\Delta s}=\lim _{\Delta s \rightarrow 0} \frac{g(z+\Delta z)-g(z)}{\Delta z} \frac{\Delta z}{\Delta s} \equiv 0,
$$

(8) The proofs of a slightly more general proposition [Walsh and Sewell 1] and [Sewell 1] seem inadequate. 
since $|\Delta z / \Delta s| \leqq 1$; it follows that $\partial g / \partial s \equiv 0$ and hence $\left({ }^{9}\right) \partial \Re[g] / \partial s=\partial \Im[g] / \partial s$ $\equiv 0$; thus $g(z)$ is a constant on $C$. Therefore

$$
f(z)=\int_{z 0}^{z} f^{\prime}(z) d z+\text { constant; }
$$

equation (2.04) follows immediately. From (2.04) we have

$$
\left|f\left(z_{2}\right)-f\left(z_{1}\right)\right| \leqq M \text { arc }\left(z_{1} \quad z_{2}\right) \leqq M A\left|z_{1}-z_{2}\right| \text {. }
$$

Theorem 2.1 yields using results due to Warschawski [1], Walsh and Sewell [1], and Sewell [1, p. 29](10)

Theorem 2.2. Let $C$ be a rectifiable Jordan curve whose arc and chord are infinitesimals of the same order. Let $f(z)$ be analytic interior to $C$, continuous on $\bar{C} ;$ let $f^{(k)}(z)$ exist in the one-dimensional sense and be continuous on $C$. Then the two-dimensional derivatives $f^{\prime}(z), f^{\prime \prime}(z), \cdots, f^{(k)}(z)$ exist on $C$, are equal to the corresponding one-dimensional derivatives on $C$, and are continuous in the two-dimensional sense on $\bar{C}$. Moreover, if $f(z) \in L_{A}(k, \alpha)\left[\right.$ or $f(z) \in \log _{A}(k, \beta)$, where $\beta=1$ or $\beta=2]$, on $C$, then $f(z) \in L_{A}(k, \alpha)\left[\right.$ or $\left.f(z) \in \log _{A}(k, \beta)\right]$, on $\bar{C}$.

We turn now to the question of the various derivatives of a harmonic function. Let $u(z)$ be harmonic interior to a rectifiable Jordan curve $C$ and continuous in $\bar{C}$. Let $h$ represent distance, considered as positive, measured from a point $z_{0}$ to a point $z_{0}+\Delta z$ on a half-line having the direction angle $\omega$ and terminating in $z_{0}$; thus we have $\Delta z=h e^{i \omega}$. If $z_{0}$ is interior to $C$, or if $z_{0}$ is on $C$ and $z_{0}+\Delta z=z_{0}+h e^{i \omega}$ lies in $\bar{C}$ for $h$ sufficiently small, then the directional derivative at $z_{0}$ of $u(z)$ in the direction $\omega$ is defined as

$$
\lim _{\Delta z \rightarrow 0} \frac{u\left(z_{0}+\Delta z\right)-u\left(z_{0}\right)}{h},
$$

provided (if $z_{0}$ is on $C$ ) this limit exists, and will be denoted by $D^{1} u\left(z_{0}\right)$. If $z_{0}$ is on $C$ and $z_{0}+\Delta z$ does not lie in $\bar{C}$ for $h$ sufficiently small, we denote by $D^{1} u\left(z_{0}\right)$

$$
\lim _{z \rightarrow z_{0}, z \text { interior to } C} D^{1} u(z),
$$

provided this limit exists. This notation fails to indicate the functional dependence of $D^{1} u(z)$ on $\omega$; all the results to be proved are valid for each $\omega$, as will appear.

Let $C$ satisfy the hypothesis of Theorem 2.2 and suppose $f(z)=u(z)+i v(z)$ is analytic interior to $C$, continuous on $\bar{C}$, and has on $C$ a continuous first

$\left({ }^{9}\right) \Re[F(z)]$ denotes the real part of $F(z) ; \Im[F(z)]$ denotes the imaginary part of $F(z)$ divided by $i$.

(10) The result needed is not there established for the case $f(z) \in \log _{A}(k, 2)$, but the proof for this case is analogous to the proofs for the other cases. 
derivative in the one-dimensional sense. Using Theorem 2.2 we obtain

$$
D^{1} u(z)=\Re\left[e^{i \omega} f^{\prime}(z)\right], \quad z \text { in } \bar{C} ;
$$

it follows that $D^{1} u(z)$ exists in $\bar{C}$ and is continuous there. Moreover an inequality of the form

$$
\left|f^{\prime}\left(z_{1}\right)-f^{\prime}\left(z_{2}\right)\right| \leqq L\left|z_{1}-z_{2}\right|^{\alpha}|\log | z_{1}-\left.z_{2}\right|^{\beta}, \quad z_{1}, z_{2} \text { on } C,
$$

implies, using Theorem 2.2,

$$
\left|D^{1} u\left(z_{1}\right)-D^{1} u\left(z_{2}\right)\right| \leqq L_{1}\left|z_{1}-z_{2}\right|^{\alpha}|\log | z_{1}-z_{2}||^{\beta}, \quad z_{1}, z_{2} \text { on } \bar{C},
$$

where either $\beta=0$ and $0<\alpha \leqq 1$, or $\alpha=\beta=1$, or $\alpha=1, \beta=2$; conversely inequality (2.07) for $z_{1}, z_{2}$ on $C$ implies (2.06) for $z_{1}, z_{2}$ on $C$ and hence on $\bar{C}$, since by taking $\omega=0$ and $\omega=\pi / 2$ in (2.05), we obtain an inequality of the form (2.07) on $\Re\left[f^{\prime}(z)\right]$ and on $\Im\left[f^{\prime}(z)\right]$, and therefore on $f^{\prime}(z)$ itself.

For $k>1$, we define $D^{k} u(z)$ as $D^{1}\left[D^{k-1} u(z)\right]$ if existent, where the successive directions $\omega_{j}, j=1, \cdots, k$, involved are arbitrary. If $C$ satisfies the hypothesis of Theorem 2.2 and $f(z)$ has a continuous $k$ th derivative on $C$ in the one-dimensional sense, then we obtain for $D^{k} u(z)$ results analogous to those obtained above for $D^{1} u(z)$. Thus we have

THEOREM 2.3. Let $C$ be a rectifiable Jordan curve whose arc and chord are infinitesimals of the same order. Let $f(z)=u(z)+i v(z)$ be analytic interior to $C$, continuous on $\bar{C} ;$ let $f^{(k)}(z), k \geqq 1$, exist in the one-dimensional sense and be continuous on $C$. Then $D^{k} u(z)$ exists and is continuous on $\bar{C}$. Furthermore, if $f^{(k)}(z)$ satisfies the condition

$$
\left|f^{(k)}\left(z_{1}\right)-f^{(k)}\left(z_{2}\right)\right| \leqq L\left|z_{1}-z_{2}\right|^{\alpha}|\log | z_{1}-z_{2}||^{\beta},
$$

for $z_{1}$ and $z_{2}$ on $C$, where $\beta=0,0<\alpha \leqq 1$, or $\alpha=\beta=1$, or $\alpha=1$ and $\beta=2$, then $D^{k} u(z)$ satisfies a corresponding condition for $z_{1}$ and $z_{2}$ on $\bar{C}$; reciprocally, if $D^{k} u(z)$ satisfies a condition of the form (2.08) on $C$, then $f^{(k)}(z)$ (still assumed existent and continuous on $C$ ) satisfies a corresponding condition on $\bar{C}$.

Finally we may consider the partial derivatives taken along $C$ :

THEOREM 2.4. Let $C$ be a rectifiable Jordan curve whose arc and chord are infinitesimals of the same order and whose equation in terms of arc-length $s$ is $z=t(s)$, where $t^{(k)}(s)$ exists and satisfies the condition

$$
\left|t^{(k)}\left(s_{1}\right)-t^{(k)}\left(s_{2}\right)\right| \leqq L\left|z_{1}-z_{2}\right|^{\alpha}|\log | z_{1}-z_{2}||^{\beta},
$$

$z_{j}=t\left(s_{j}\right)$, where either $\beta=0$ and $0<\alpha \leqq 1$, or $\alpha=\beta=1$, or $\alpha=1$ and $\beta=2$. Let the function $f(z)=u(z)+i v(z)$ be defined on $C$. If $f^{(k)}(z)$ exist and satisfies a condition of the form (2.08) on $C$, then $\partial^{k} f / \partial s^{k}, \partial^{k} u / \partial s^{k}$ exists on $C$ and satisfy on $C$ corresponding conditions. Conversely, if $\partial^{k} f / \partial s^{k}$ exists and satisfies a condition of the form (2.08) on $C$, then $f^{(k)}(z)$ exists and satisfies a condition of the form (2.08) on $C$. 
The proof follows from Theorem 2.1 and the equations

$$
\begin{aligned}
& \frac{\partial f}{\partial s}=f^{\prime}(z) \cdot t^{\prime}(s), \quad \frac{\partial^{2} f}{\partial s^{2}}=f^{\prime \prime}(z)\left[t^{\prime}(s)\right]^{2}+f^{\prime}(z) \cdot t^{\prime \prime}(s), \\
& \frac{\partial^{3} f}{\partial s^{3}}=f^{\prime \prime \prime}(z)\left[t^{\prime}(s)\right]^{3}+3 f^{\prime \prime}(z) \cdot t^{\prime \prime}(s) \cdot t^{\prime}(s)+f^{\prime}(z) \cdot t^{\prime \prime \prime}(s),
\end{aligned}
$$

and so on.

3. Lipschitz conditions and continuity. Relating the various classes of functions defined in $\$ 2$ we prove

THEOREM 3.1. Let $u(z) \in L(k, \alpha)$ on $|z|=1$. Set $f(z)=u(z)+i v(z)$, where $v(z)$ is conjugate $\left({ }^{11}\right)$ to $u(z)$ in $|z| \leqq 1, v(0)=0$. Then if $0<\alpha<1, f(z) \in L_{A}(k, \alpha)$ on $|z| \leqq 1$; if $\alpha=1, f(z) \in \log _{A}(k, 1)$ on $|z| \leqq 1$. If $u(z) \in \log (k, 1)$ on $|z|=1$, then $f(z) \in \log _{A}(k, 2)$ on $|z| \leqq 1$.

For the case $u(z) \in L(0, \alpha)$ on $|z|=1$, Privaloff [1] (for example, Zygmund $[1, \mathrm{pp} .156-157])$ has proved that $v(z) \in L(0, \alpha)$ on $|z|=1$ if $0<\alpha<1$ and $v(z) \in \log (0,1)$ on $|z|=1$ if $\alpha=1$. If $u(z) \in \log (0,1)$ on $|z|=1$, it can be shown by Privaloff's method that $v(z) \in \log (0,2)$ on $|z|=1$. The theorem thus follows for $k=0$ thanks to Theorem 2.2.

Suppose $k>0$. We shall prove that $\partial^{k} v\left(r e^{i \theta}\right) / \partial \theta^{k}, z=r e^{i \theta}$, is conjugate to $\partial^{k} u\left(r e^{i \theta}\right) / \partial \theta^{k},|z| \leqq 1$. It is clearly sufficient to establish this result for the case $k=1$, since the result for $k>1$ will then follow by iteration. If $a_{j}, b_{j}$ are the Fourier coefficients of $u\left(e^{i \theta}\right), 0 \leqq \theta \leqq 2 \pi$, we have for $|z| \leqq 1$

$$
\begin{aligned}
& u\left(r e^{i \theta}\right)=\left(a_{0} / 2\right)+\sum_{j=1}^{\infty} r^{j}\left(a_{j} \cos j \theta+b_{j} \sin j \theta\right), \\
& v\left(r e^{i \theta}\right)=\sum_{j=1}^{\infty} r^{j}\left(a_{j} \sin j \theta-b_{j} \cos j \theta\right) .
\end{aligned}
$$

Hence by the usual equations for the Fourier coefficients of the derivative, we have the expansion

$$
\frac{\partial u\left(r e^{i \theta}\right)}{\partial \theta}=\sum_{j=1}^{\infty} r^{j}\left(-j a_{j} \sin j \theta+j b_{j} \cos j \theta\right),
$$

converging uniformly in $|z| \leqq 1$. Thus the function conjugate to $\partial u\left(r e^{i \theta}\right) / \partial \theta$ is

$$
g\left(r e^{i \theta}\right)=\sum_{j=1}^{\infty} r^{j}\left(j a_{j} \cos j \theta+j b_{j} \sin j \theta\right) ;
$$

(11) If two functions are conjugate in a region and continuous in the corresponding closed region, we say that the functions are conjugate in the closed region. 
since by Privaloff's results $g\left(e^{i \theta}\right)$ satisfies a Lipschitz condition of positive order, the right side of (3.01) converges uniformly on $|z| \leqq 1$. From the classical theorem on term-by-term differentation of a series of the real variable $\theta$, where $r$ is considered fixed $(0<r \leqq 1)$, it follows that $\partial v\left(r e^{i \theta}\right) / \partial \theta$ exists and equals $g\left(r e^{i \theta}\right)$.

Theorem 3.1 now follows for $k>0$ by Theorem 2.4 and Privaloff's results.

By the use of conformal mapping Theorem 3.1 extends to curves more general than the unit circle and, in particular, to analytic Jordan curves.

\section{Part I. ApProximation on the Unit CIRCle}

4. Problem $\alpha$. By a harmonic polynomial $p_{n}(x, y)=p_{n}(z)$ of degree $n$ we mean a harmonic function of the form

$$
p_{n}(z)=\sum_{0 \leqq i+j \leqq n} c_{i, j} x^{i} y^{j}, \quad i, j \text { non-negative integers, }
$$

where the $c_{i, j}$ are real. The degree of a polynomial will always be indicated by its subscript. We do not assume $\sum\left|c_{i, j}\right|$, where $i+j=n$, to be different from zero; thus a polynomial of degree $m, 0 \leqq m<n$, is also a polynomial of degree $n$. If $q_{n}(z)$ is conjugate to $p_{n}(z), q_{n}(0)=0$, then

$$
q_{n}\left(z_{0}\right)=\int_{0}^{z_{0}}\left(\frac{\partial p_{n}(x, y)}{\partial x} d y-\frac{\partial p_{n}(x, y)}{\partial y} d x\right),
$$

the integral being taken along the broken line $y=0,0 \leqq x \leqq x_{0} ; x=x_{0}, 0 \leqq y \leqq y_{0}$, where $z_{0}=x_{0}+i y_{0}$. Hence $q_{n}(z)$ is also a harmonic polynomial of degree $n$. Clearly $\pi_{n}(z)=p_{n}(z)+i q_{n}(z)$ is a polynomial in $z$ of degree $n$, for

$$
\pi^{(n+1)}(z)=\left(\partial^{n+1} p_{n} / \partial x^{n+1}\right)+i\left(\partial^{n+1} q_{n} / \partial x^{n+1}\right) \equiv 0 .
$$

Thus since $p_{n}(z)=\Re\left[\pi_{n}(z)\right], p_{n}(z)$ may be expressed as

$$
p_{n}(z)=\sum_{j=0}^{n}\left(a_{j} \Re\left[z^{j}\right]+b_{j} \Im\left[z^{j}\right]\right),
$$

where $a_{j}, b_{j}, j=0,1, \cdots, n$, are real. Conversely, if $\pi_{n}(z)$ is a polynomial in $z$ of degree $n, \Re\left[\pi_{n}(z)\right]$ and $\Im\left[\pi_{n}(z)\right]$ are harmonic polynomials of degree $n$.

If polar coordinates $(r, \theta)$ are introduced in the $z$-plane, the problem of approximation on the unit circle by harmonic polynomials becomes a problem in trigonometric approximation. A function of the form

$$
\sum_{k=0}^{n} r^{k}\left(a_{k} \cos k \theta+b_{k} \sin k \theta\right)
$$

where $a_{k}, b_{k}$ are real, is a harmonic polynomial of degree $n$ in $x$ and $y$, for $r^{k} \cos k \theta=\Re\left[z^{k}\right], r^{k} \sin k \theta=\Im\left[z^{k}\right]$; conversely, a harmonic polynomial of degree $n$ in $x$ and $y$ can be written as 


$$
\sum_{k=0}^{n}\left(\alpha_{k} \Re\left[z^{k}\right]+\beta_{k} \Im\left[z^{k}\right]\right)=\sum_{k=0}^{n}\left(\alpha_{k} r^{k} \cos k \theta+\beta_{k} r^{k} \sin k \theta\right),
$$

which is of the form (4.01). On $\gamma:|z|=1$ a function (4.01) is a trigonometric sum of order $n$; conversely a trigonometric sum of order $n$ may be regarded as the values on $\gamma$ of a function (4.01).

Let $u(z) \in L(k, \alpha)$ on $\gamma$. It follows from Jackson [1] that for each $n$, $n=1,2, \cdots$, there exists a trigonometric sum $T_{n}(\theta)$ of order $n$ such that

$$
\left|u\left(e^{i \theta}\right)-T_{n}(\theta)\right| \leqq M / n^{k+\alpha}
$$

By the principle of the maximum for harmonic functions we thus have

Theorem 4.1. Let $u(z) \in L(k, \alpha)$ on $\gamma:|z|=1,0<\alpha \leqq 1$. Then there exist harmonic polynomials $p_{n}(z)$ such that

$$
\left|u(z)-p_{n}(z)\right| \leqq M / n^{k+\alpha}, \quad|z| \leqq 1 .
$$

Here and throughout we write "there exist polynomials" rather than "for each $n, n=1,2, \cdots$, there exists a polynomial of degree $n$."

If $p_{n}(z)$ is defined as the sum of the first $n+1$ terms of the Fourier development of $u\left(e^{i \theta}\right)$, inequality (4.02) is valid if the second member is replaced by $M_{1} \log n / n^{k+\alpha}$ (see Jackson [1, p. 22]).

We turn now to the converse question. Let $U(\theta)$ be defined for all $\theta$, and periodic with period $2 \pi$. De la Vallée Poussin [1, Chap. IV] has shown that if there exist trigonometric sums $T_{n}(\theta)$ of order $n$ such that

$$
\left|U(\theta)-T_{n}(\theta)\right| \leqq M / n^{k+\alpha}, \quad 0<\alpha \leqq 1 .
$$

then $U^{(k)}(\theta)$ exists and satisfies the condition

$$
\left|U^{(k)}\left(\theta_{1}\right)-U^{(k)}\left(\theta_{2}\right)\right| \leqq M_{1}\left|\theta_{1}-\theta_{2}\right|^{\alpha}|\log | \theta_{1}-\theta_{2}||^{\beta},
$$

where $\beta=0$ if $\alpha<1, \beta=1$ if $\alpha=1$. We thus have by the definitions of $L(k, \alpha)$ and $\log (k, 1)$

THEOREM 4.2. Let $u(z)$ be defined in $|z| \leqq 1$. Let there exist harmonic polynomials $p_{n}(z)$ such that

$$
\left|u(z)-p_{n}(z)\right| \leqq M / n^{k+\alpha}, \quad|z| \leqq 1 .
$$

If $0<\alpha<1$, then $u(z) \in L(k, \alpha)$ on $|z|=1$; if $\alpha=1$, then $u(z) \in \log (k, 1)$ on $|z|=1$.

5. Problem $\beta$. We shall investigate in this section the relationship between continuity properties of $u(z)$ on $|z|=R(>1)$, where $u(z)$ is assumed harmonic in $|z|<R$, and degree of approximation to $u(z)$ on $|z| \leqq 1$.

THEOREM 5.1. Let $u(z)$ be harmonic in $|z|<R(>1)$, continuous in $|z| \leqq R$; 
suppose there exists a harmonic polynomial $p_{n}(z)$ such that

$$
\int_{|z|=R}\left|u(z)-p_{n}(z)\right|^{p}|d z| \leqq \underset{\epsilon_{n},}{p}, \quad p>1
$$

Then for the sum $S_{n}(z)$ of the first $n+1$ terms of the Fourier expansion

$$
u\left(r e^{i \theta}\right)=\left(a_{0} / 2\right)+\sum_{j=1}^{\infty} r^{i}\left(a_{j} \cos j \theta+b_{j} \sin j \theta\right), \quad r<R,
$$

we have

$$
\left|u(z)-S_{n}(z)\right| \leqq M \epsilon_{n} / R^{n}, \quad|z| \leqq 1 .
$$

Using Poisson's integral and the orthogonality properties of $\sin j \theta$ and $\cos j \theta$, we obtain for $1<\rho<R$

$$
\begin{aligned}
u\left(e^{i \theta}\right)-S_{n}\left(e^{i \theta}\right)=\frac{1}{2 \pi} \int_{0}^{2 \pi} \sum_{j=n+1}^{\infty} \rho^{j}\left(a_{j} \cos j \psi+b_{j} \sin j \psi\right) & \quad\left(1+2 \sum_{\mu=1}^{\infty} \rho^{-\mu} \cos \mu(\theta-\psi)\right) d \psi \\
& =\frac{1}{\pi} \int_{0}^{2 \pi} u\left(\rho e^{i \psi}\right)\left(\sum_{\mu=n+1}^{\infty} \rho^{-\mu} \cos \mu(\theta-\psi)\right) d \psi \\
& =\frac{1}{\pi} \int_{0}^{2 \pi}\left[u\left(\rho e^{i \psi}\right)-p_{n}\left(\rho e^{i \psi}\right)\right]\left[\sum_{\mu=n+1}^{\infty} \rho^{-\mu} \cos \mu(\theta-\psi)\right] d \psi .
\end{aligned}
$$

Use of Hölder's inequality yields

$$
\left|u\left(e^{i \theta}\right)-S_{n}\left(e^{i \theta}\right)\right| \leqq 2 \rho^{-n}(\rho-1)^{-1}\left[\int_{0}^{2 \pi}\left|u\left(\rho e^{i \psi}\right)-p_{n}\left(\rho e^{i \psi}\right)\right|^{p} d \psi\right]^{1 / p} .
$$

The theorem now follows on allowing $\rho$ to approach $R$.

Theorems 4.1 and 5.1 yield

Theorem 5.2. Let $u(z) \in L(k, \alpha), 0<\alpha \leqq 1$, on $|z|=R, R>1$. Then for the sum $S_{n}(z)$ of the first $n+1$ terms of the expansion

$$
u\left(r e^{i \theta}\right)=\left(a_{0} / 2\right)+\sum_{j=1}^{\infty} r^{i}\left(a_{j} \cos j \theta+b_{j} \sin j \theta\right), \quad r<R,
$$

we have

$$
\left|u(z)-S_{n}(z)\right| \leqq L / n^{k+\alpha} R^{n}, \quad|z| \leqq 1 .
$$

We prove a theorem related to Theorems 5.1 and 5.2:

THeORem 5.3. Let $u(z)$ be harmonic in $|z|<R(>1)$, continuous in $|z| \leqq R$, 
with the development for $|z| \leqq R$

$$
u(z)=\lim _{n \rightarrow \infty} P_{n}(z), \quad P_{n}(z)=\sum_{j=0}^{n} r^{i}\left(a_{j} \cos j \theta+b_{j} \sin j \theta\right) .
$$

If $\left|u(z)-P_{n}(z)\right| \leqq \epsilon_{n},|z|=R$, where $\epsilon_{n}$ approaches zero monotonically with $1 / n$, then

$$
\left|u(z)-P_{n}(z)\right| \leqq 2 \epsilon_{n} / R^{n+1}, \quad|z|=1 .
$$

From the hypothesis we may write for $m>n$

$$
\left|P_{m}(z)-P_{n}(z)\right| \leqq 2 \epsilon_{n}, \quad|z|=R .
$$

Abel's Lemma then yields

$$
\left|\sum_{j=n+1}^{m}\left(a_{i} \cos j \theta+b_{i} \sin j \theta\right)\right| \leqq 2 \epsilon_{n} / R^{n+1} .
$$

Theorem 5.3 follows by allowing $m$ to become infinite.

We turn now to the converse problem. The following theorem due to Szegö [1] (see also Schaeffer and Szegö [1]) will be useful:

THEOREM 5.4. Let $p_{n}(z)$ be a harmonic polynomial of degree $n$. If $\left|p_{n}(z)\right| \leqq K$ on $|z|=1$, then

$$
\left|p_{n}(z)\right| \leqq K R^{n} \quad \text { on }|z| \leqq R, R>1 .
$$

Using this theorem we prove

THEOREM 5.5. Let $u(z)$ be defined on $|z| \leqq 1$. Let harmonic polynomials $p_{n}(z)$ exist such that

$$
\left|u(z)-p_{n}(z)\right| \leqq M / n^{k+\alpha+1} R^{n}, \quad|z| \leqq 1, R>1 .
$$

Then when $u(z)$ is suitably defined $\left({ }^{12}\right)$ exterior to $|z| \leqq 1, u(z) \in L(k, \alpha)$ on $|z|=R$ if $0<\alpha<1, u(z) \in \log (k, 1)$ on $|z|=R$ if $\alpha=1$.

We have for the function $u(z)$ the representation

$$
u(z)=p_{1}(z)+\left[p_{2}(z)-p_{1}(z)\right]+\left[p_{3}(z)-p_{2}(z)\right]+\cdots .
$$

Use of the triangle inequality yields from (5.01)

$$
\left|p_{n+1}(z)-p_{n}(z)\right| \leqq 2 M / n^{k+\alpha+1} R^{n}, \quad|z| \leqq 1 .
$$

Hence by Theorem 5.4

$$
\left|p_{n+1}(z)-p_{n}(z)\right| \leqq M_{1} / n^{k+\alpha+1}, \quad|z| \leqq R .
$$

(12) Here and in following similar situations we assume that if $u(z)$ or $f(z)$ is not originally supposed to be defined on the entire point set considered, the supplementary definition is to be made by means of the convergent series of polynomials, which is equivalent to defining $u(z)$ by harmonic continuation and $f(z)$ by analytic continuation. 
Thus

$$
\left|u(z)-p_{n}(z)\right| \leqq M_{1} \sum_{\nu=n}^{\infty} \nu^{-(k+\alpha+1)} \leqq M_{2} / n^{k+\alpha}, \quad|z| \leqq R .
$$

Theorem 5.5 now follows from Theorem 4.2.

Theorem 5.5. may also be proved by means of

THEOREм 5.6. Let $u(z)$ be continuous on $|z|=1$. Suppose there exist harmonic polynomials $p_{n}(z)$ such that

$$
\int_{0}^{2 \pi}\left|u\left(e^{i \theta}\right)-p_{n}\left(e^{i \theta}\right)\right|^{p} d \theta=\epsilon_{n}^{p} / R^{p n}, \quad R>1, p>1,
$$

or more generally suppose the nth order Fourier coefficients of $u\left(e^{i \theta}\right)$ not greater in absolute value than $M_{1} \epsilon_{n-1} / R^{n-1}$. If $\sum \epsilon_{j}$ converges, then $u(z)$ can be defined $\left.{ }^{18}\right)$ so as to be harmonic in the region $|z|<R$, continuous in $|z| \leqq R$; on $|z|=R, u(z)$ can be approximated by trigonometric sums of respective degrees $n$ with an error of approximation not greater than $M \sum_{j=n}^{\infty} \epsilon_{j}$.

The required trigonometric sums can be taken as the partial sums of the formal development

$$
u(z)=\sum_{j=0}^{\infty} r^{i}\left(a_{j} \cos j \theta+b_{j} \sin j \theta\right)
$$

where $a_{j}, b_{j}$ are the Fourier coefficients of $u\left(e^{i \theta}\right)$. Then

$$
\begin{aligned}
a_{j+1} & =\frac{1}{\pi} \int_{0}^{2 \pi} u\left(e^{i \theta}\right) \cos (j+1) \theta d \theta \\
& =\frac{1}{\pi} \int_{0}^{2 \pi}\left[u\left(e^{i \theta}\right)-p_{j}\left(e^{i \theta}\right)\right] \cos (j+1) \theta d \theta .
\end{aligned}
$$

Thus by Hölder's inequality and (5.02), if (5.02) is assumed, and otherwise by hypothesis, we have $\left|a_{j+1}\right| \leqq M_{2} \epsilon_{j} / R^{j},\left|b_{j+1}\right| \leqq M_{2} \epsilon_{j} / R^{i}$. In any case equation (5.03) defines $u(z)$ as a function harmonic for $|z|<R$, continuous for $|z| \leqq R$, such that we have on $|z|=R$ the desired inequality

$$
\left|u(z)-\sum_{j=0}^{n} R^{i}\left(a_{j} \cos j \theta+b_{j} \sin j \theta\right)\right| \leqq M_{3} \sum_{j=n}^{\infty} \epsilon_{j} .
$$

6. Problem $\beta$, continued. As a preliminary to general remarks on trigonometric approximation we mention

${ }^{(13)}$ Originally $u(z)$ appears merely on the circle $|z|=1$, and there only by means of an integral; hence $u(z)$ need not even be continuous on $|z|=1$ unless that requirement is made explicitly. 
THEOREM 6.1. If for the series

$$
\frac{a_{0}}{2}+\sum_{n=1}^{\infty} r^{n}\left(a_{n} \cos n \theta+b_{n} \sin n \theta\right)
$$

we set $\left(z=r e^{i \theta}\right)$

$$
\limsup _{n \rightarrow \infty}\left[a_{n}^{2}+b_{n}^{2}\right]^{1 / 2 n}=1 / \rho
$$

where $\rho$ is finite or infinite, then the series (6.01) converges uniformly on every circle $|z|=\rho^{\prime}<\rho$; it does not converge at every point of any arc of a circle $|z|=\rho^{\prime \prime}>\rho$.

The uniform convergence of (6.01) on $|z|=\rho^{\prime}$ follows immediately from the well known theorem on the uniform convergence on $|z|=\rho^{\prime}$ of the power series $a_{0} / 2+\sum_{n=1}^{\infty}\left(a_{n}-i b_{n}\right) z^{n}$, of which (6.01) is the real part.

On the other hand, if $r=\rho^{\prime \prime}$ we have

$$
\underset{n \rightarrow \infty}{\limsup }\left[a_{n}^{2} r^{2 n}+b_{n}^{2} r^{2 n}\right]^{1 / 2 n}=\rho^{\prime \prime} / \rho>1 \text {; }
$$

hence the coefficients $a_{n} r^{n}$ and $b_{n} r^{n}$ do not tend to zero with $1 / n$, and the second part of the theorem follows from the Cantor theorem on the coefficients of a trigonometrical series.

In the general study of convergence of the series

$$
\frac{a_{0}}{2}+\sum_{n=1}^{\infty}\left(a_{n} \cos n \theta+b_{n} \sin n \theta\right)
$$

it is natural to relate the degree of convergence of (6.03) to the continuity properties of the function represented. But if the number $\rho$ defined by (6.02) is greater than unity, the function defined by (6.03) is an analytic function of $\theta$, so that all the usual requirements of continuity of both the function and its derivatives are automatically fulfilled. We should like then to associate with the function (6.03) some such function as (6.01), and to relate the degree of convergence of (6.03) to the continuity properties of (6.01). There are various possibilities here, all under the assumption that $\rho$ is greater than unity.

I. We may set $z=r e^{i \theta}$ and consider (6.03) as the values on the circle $|z|=1$ of the function

$$
\sum_{n=-\infty}^{\infty} c_{n} z^{n}, \quad 2 c_{n}=a_{n}-i b_{n}, \quad c_{-n}=\bar{c}_{n}, \quad n \geqq 0 .
$$

We are then in a position to relate the degree of convergence of (6.03) to the continuity properties of (6.04) on the circles of convergence $|z|=\rho$ and $|z|=1 / \rho$. This study will not be treated here but has already been undertaken by de la Vallée Poussin [1] in the case that (6.04) has poles on the 
circles of convergence, and by Walsh and Sewell $[3,4]$ in the case that (6.04) is continuous on these circles.

II. We may study the connection between degree of convergence of (6.03) and the continuity properties of the harmonic function (6.01) on the circle $|z|=\rho$; we have already done this $(\$ 5)$.

III. We can study the relation between degree of convergence of (6.03) and continuity properties on $|z|=1 / \rho$ of the harmonic function

$$
\frac{a_{0}}{2}+\sum_{n=1}^{\infty} r^{-n}\left(a_{n} \cos n \theta+b_{n} \sin n \theta\right)
$$

This problem is essentially the same as II, for the functions (6.01) and (6.05) are transforms of each other under the transformation $z=1 / \bar{z}$.

IV. We can identify (6.03) with the series

$$
\sum_{n=-\infty}^{\infty} r^{n}\left(a_{n}^{\prime} \cos n \theta+b_{n}^{\prime} \sin n \theta\right)
$$

on the circle $|z|=1$, where the coefficients are chosen to satisfy

$$
a_{n}^{\prime}+a_{-n}^{\prime}=a_{n}, \quad b_{n}^{\prime}-b_{-n}^{\prime}=b_{n}, \quad n \geqq 0 .
$$

Equations (6.07) obviously do not determine $a_{n}^{\prime}$ and $b_{n}^{\prime}$ uniquely, and there is perhaps no "best" choice. Nevertheless, when (6.03) is given, to choose $a_{n}^{\prime}$ and $b_{n}^{\prime}$ for $n>0$ in such a way that

$$
\limsup _{n \rightarrow \infty}\left[{a_{n}^{\prime 2}}^{2}+{b_{n}^{\prime 2}}^{1 / 2 n}>1 / \rho\right.
$$

is obviously unfavorable, for under those circumstances the series

$$
\sum_{n=1}^{\infty} r^{n}\left(a_{n}^{\prime} \cos n \theta+b_{n}^{\prime} \sin n \theta\right)
$$

does not even converge throughout the interior of the circle $|z|=\rho$, and Theorems 5.1, 5.2, 5.3 yield weaker results than those obtained by methods II and III. Similarly it is unfavorable to choose $a_{n}^{\prime}$ and $b_{n}^{\prime}$ for $n<0$ in such a way that

$$
\limsup _{n \rightarrow \infty}\left[a_{-n}^{\prime 2}+b_{-n}^{\prime 2}\right]^{1 / 2 n}>1 / \rho
$$

Since such reasoning as used in Theorems 5.1, 5.2, and 5.3 yields degree of convergence depending on the weaker of the continuity properties of (6.08) on $|z|=\rho$ and

$$
\sum_{n=-\infty}^{0} r^{n}\left(a_{n}^{\prime} \cos n \theta+b_{n}^{\prime} \sin n \theta\right)
$$


on $|z|=1 / \rho$, it is unfavorable to choose $a_{n}^{\prime}$ and $b_{n}^{\prime}, n>0$, in such a way that the continuity properties of (6.08) on $|z|=\rho$ are either better or worse than those of (6.09) on $|z|=1 / \rho$.

Thus possible and natural choices of $a_{n}^{\prime}$ and $b_{n}^{\prime}$ in (6.07) correspond to expressing (6.03) as the sum of a suitable multiple of (6.01) and a suitable multiple of (6.05) on the circle $|z|=1$. The most natural choice other than those mentioned in II and III would seem to be

$$
a_{n}^{\prime}=a_{-n}^{\prime}=a_{n} / 2, \quad b_{n}^{\prime}=-b_{-n}^{\prime}=b_{n} / 2, \quad n \geqq 0 .
$$

This choice corresponds to using method II for half of the function (6.06), using method III for the other half of that function, and combining the results. The final conclusions on degree of convergence are then essentially those obtained by method II.

\section{Part II. Approximation on more general SETS}

7. Polynomial inequalities. To aid in the establishment of further theorems for Problem $\alpha$, we shall prove certain results on harmonic polynomials based on the following theorem due to Szegö $\left({ }^{14}\right)$ :

TheOREM 7.1. Let $\pi_{n}(z)$ be a polynomial in $z$ of degree $n$ such that $\left|\Re\left[\pi_{n}(z)\right]\right|$ $\leqq K$ for $\left|z-z_{0}\right| \leqq R$. Then

$$
\left|\pi_{n}^{\prime}(z)\right| \leqq n K / R \quad \text { for }\left|z-z_{0}\right| \leqq R
$$

We make

Definition 7.1. A curve $C$ is said to be of Type D if

(i) $C$ is a rectifiable Jordan curve whose arc and chord are infinitesimals of the same order; and

(ii) there exists a number $\delta_{0}>0$ such that if $P$ is any point of $C$, there exists a circle of radius $\delta_{0}$ through $P$ whose closed interior lies in $\bar{C}$.

Seidel [1] has proved that if $C$ possesses a tangent at each point and is such that $|\omega(s+h)-\omega(s)| \leqq L_{0} h, h \geqq 0$, where $\omega$ denotes the angle from the axis of reals to the positively directed tangent to $C$, and $s$ denotes arc-length measured along $C$, then $C$ satisfies (ii).

From Theorem 7.1 we obtain easily

Theorem 7.2. Let $C$ be a curve of Type D. Let $\pi_{n}(z)$ be a polynomial in $z$ of degree $n$. If $\left|\Re\left[\pi_{n}(z)\right]\right| \leqq K, z$ on $C$, then

$$
\left|\pi_{n}^{\prime}(z)\right| \leqq K n / \delta_{0}, \quad \quad z \text { on } \bar{C},
$$

where $\delta_{0}$ is the constant which occurs in Definition 7.1.

Under the hypothesis on $C$, if $P$ is any point of $C$, there exists a circle $\gamma_{P}$

${ }^{\left({ }^{14}\right)}$ Szegö [2] proves the theorem for the case of the unit circle, but the extension to an arbitrary circle follows at once. 
through $P$, radius $\delta_{0}$, lying entirely in $\bar{C}$. In each such circle $\left|\Re\left[\pi_{n}(z)\right]\right| \leqq K$. Therefore by Theorem 7.1, $\left|\pi_{n}^{\prime}(z)\right| \leqq K n / \delta_{0}$ for $z$ in $\bar{\gamma}_{P}$. Since this last inequality holds for all $z$ on $C$, it holds for $z$ on $\bar{C}$.

Corollary 7.3. Let $C$ be a curve of Type D. If $p_{n}(z)$ is a harmonic polynomial of degree $n$ such that $\left|p_{n}(z)\right| \leqq K, z$ on $C$, then

$$
\left|\operatorname{grad} p_{n}(z)\right| \leqq K n / \delta_{0}, \quad \quad z \text { in } \bar{C} .
$$

Theorem 7.4. Let $C$ be a curve of Type D. Let $\pi_{n}(z)$ be a polynomial in $z$ of degree $n$, and set $\Re\left[\pi_{n}(z)\right]=p_{n}(z)$. If $\left|p_{n}(z)\right| \leqq K, z$ on $C$, then

$$
\left|p_{n}\left(z_{1}\right)-p_{n}\left(z_{2}\right)\right| \leqq\left|\pi_{n}\left(z_{1}\right)-\pi_{n}\left(z_{2}\right)\right| \leqq A K n\left|z_{1}-z_{2}\right| / \delta_{0}, \quad z_{1}, z_{2} \text { on } \bar{C},
$$

where $A$ and $\delta_{0}$ are the constants of Definitions 2.1 and 7.1, respectively.

By Theorem 7.2, $\left|\pi_{n}^{\prime}(z)\right| \leqq K n / \delta_{0}, z$ on $C$; thus Theorem 7.4 follows from Theorem 2.1 and Sewell [1, Theorem 1.2.7].

Theorem 7.5. Let $C$ be a curve of Type $\mathrm{D}$, and let $z_{0}$ be a point interior to $C$. Suppose $\pi_{n}(z)=p_{n}(z)+i q_{n}(z), q_{n}\left(z_{0}\right)=0$, is a polynomial in $z$ of degree $n>1$. If $\left|p_{n}(z)\right| \leqq K, z$ on $C$, then

$$
\left|\pi_{n}(z)\right| \leqq L K \log n, \quad \text { zon } \bar{C} .
$$

Let $C^{\prime}$ be an analytic Jordan curve interior to $C$ which contains in its interior $z_{0}$ and each point of $\bar{C}$ situated at a distance from $C$ greater than $\delta_{0} / 2$, where $\delta_{0}$ is the constant of Definition 7.1. Then we have [Walsh 5, p. 323] $\left|q_{n}(z)\right| \leqq L_{1} K, z$ in $\bar{C}^{\prime}$.

Let $z$ be any point on $C$ and let $\zeta$ be the center of a circle through $z$ with radius $\delta_{0}$ which lies entirely in $\bar{C}$. Then by a theorem due to Fekete $[1$, p. 21] (see also Carleman $\left[1\right.$, p. 383]) we have $\left|q_{n}(z)-q_{n}(\zeta)\right| \leqq L_{2} K \log n$. Since $\zeta$ lies in $\bar{C}^{\prime}$, it follows for $z$ on $C$ and hence for $z$ on $\bar{C}$ that

$$
\begin{aligned}
\left|\pi_{n}(z)\right| & \leqq\left|p_{n}(z)\right|+\left|q_{n}(z)-q_{n}(\zeta)\right|+\left|q_{n}(\zeta)\right| \leqq K+L_{2} K \log n+L_{1} K \\
& \leqq L K \log n .
\end{aligned}
$$

8. Problem $\alpha$. By using a modification of a method due to Curtiss [1] it is possible to extend Theorem 4.1 to more general sets. If $C$ consists of a finite number of Jordan curves, we denote by $\bar{C}$ the sum of their closed interiors.

THEOREM 8.1. Let $C$ consist of a finite number of mutually exterior analytic Jordan curves. Let $u(z) \in L(k, \alpha)$ on $C, 0<\alpha \leqq 1$. Set $f(z)=u(z)+i v(z)$, where $v(z)$ is conjugate to $u(z)$ in $\bar{C}$. Then there exist polynomials $\pi_{n}(z)$ in $z$ such that

$$
\left|f(z)-\pi_{n}(z)\right| \leqq M / n^{k+\alpha}, \quad z \text { in } \bar{C} .
$$

If $0<\alpha<1$, it follows ( $\$ 3)$ that $f(z) \in L_{A}(k, \alpha)$ on $C$, which implies the conclusion at once (see, for example, Sewell [1, Theorem 3.2.1]).

Now consider the case $\alpha=1$. Suppose $C$ consists of the Jordan curves 
$C_{1}, C_{2}, \cdots, C_{\lambda}$. Let $w=\Omega_{\nu}(z)$ map the interior of $C_{\nu}$ one-to-one and conformally onto the interior of $\gamma:|w|=1$; denote the inverse mapping function by $z=\chi_{\nu}(w)$. Since the curves $C_{\nu}, \nu=1, \cdots, \lambda$, are analytic and mutually exterior, there exists a number $R>1$ such that the curves $C_{\nu}^{\prime}:\left|\Omega_{\nu}(z)\right|=R$ are analytic and mutually exterior and such that the functions $\Omega_{\nu}(z)$ are analytic and schlicht in $\bar{C}_{\nu}^{\prime}$, respectively.

By the analyticity of the $C_{\nu}, u\left[\chi_{\nu}(w)\right] \in L(k, \alpha)$ on $\gamma$. Since $\partial^{k} v\left[\chi_{\nu}\left(r e^{i \theta}\right)\right] / \partial \theta^{k}$ is conjugate (cf. $\S 3$ ) to $\partial^{k} u\left[\chi_{\nu}\left(r e^{i \theta}\right)\right] / \partial \theta^{k}$ in $|w| \leqq 1, w=r e^{i \theta}$, it therefore follows from results due to Zygmund [2, Theorem 7 and §5] that

$$
\left|f\left[\chi_{\nu}\left(e^{i \theta}\right)\right]-\sum_{\mu=0}^{m} d_{m, \mu}\left(a_{\nu, \mu}-i b_{\nu, \mu}\right) e^{i \mu \theta}\right| \leqq M_{0} / m^{k+1},
$$

where $a_{\nu, \mu}, b_{\nu, \mu}$ are the Fourier coefficients of $u\left[\chi_{\nu}\left(e^{i \theta}\right)\right]$ and $d_{m, \mu}$ are the Jackson summation coefficients of order $k$; compare also Sewell [1, $\$ 3.1$ and 3.2]. Hence there exist polynomials in $w: \pi_{\nu, m}(w)=\sum_{\mu=0}^{m} d_{m, \mu}\left(a_{\nu, \mu}-i b_{\nu, \mu}\right) w^{\mu}$ $=F_{\nu, m}(z)$ such that

$$
\left|f(z)-F_{\nu, m}(z)\right| \leqq M_{1} / m^{k+1}, \quad z \text { on } \bar{C}_{\nu}, \nu=1, \cdots, \lambda .
$$

By (8.01) the polynomials $\pi_{v, m}(w)$ are uniformly bounded on $|w|=1$; hence (see, for example, Walsh [4, p. 77])

$$
\left|F_{\nu, m}(z)\right| \leqq M_{2} R^{m}
$$

$z$ on $C_{\nu}^{\prime}$.

Define $F_{m}(z)=F_{\nu, m}(z), z$ in $\bar{C}_{\nu}^{\prime}, \nu=1, \cdots, \lambda$. Let $w=\phi(z)$ denote a function which maps the exterior of $C$ conformally (not necessarily uniformly) onto the exterior of $|w|=1$ so that the points at infinity in the two planes correspond to each other. That is to say, we set $\phi(z)=e^{o(z)+i h(z)}$, where $g(z)$ is Green's function for the exterior of $C$ with pole at infinity and $h(z)$ is conjugate to $g(z)$; compare for instance Walsh [4, pp. $65 \mathrm{ff}$.]. Let $\mu>1$ be a number such that $\Gamma:|\phi(z)|=\mu$ consists of $\lambda$ analytic Jordan curves lying respectively interior to the curves $C_{\nu}^{\prime}$ and containing the curves $C_{\nu}$ in their respective interiors; such a number exists by virtue of the analyticity of each $C_{\nu}$.

Let $P_{n}(z)$ be the polynomial in $z$ of degree $n$ which interpolates to $F_{m}(z)$ in $n+1$ points $z_{j}$ equally distributed $\left({ }^{15}\right)$ on $C$; then we have in the usual notation

$$
F_{m}(z)-P_{n}(z)=\frac{1}{2 \pi i} \int_{\Gamma} \frac{\omega_{n}(z) F_{m}(t)}{\omega_{n}(t)(t-z)} d t,
$$

where $\omega_{n}(z)=\left(z-z_{1}\right)\left(z-z_{2}\right) \cdots\left(z-z_{n+1}\right)$. It has been shown [Walsh and Sewell 1] that $\left|\omega_{n}(z) / \omega_{n}(t)\right| \leqq M_{3} / \mu^{n}$ for $z$ on $C$ and $t$ on $\Gamma$. Hence by (8.02)

(15) See Walsh and Sewell [1] for details of this paragraph. The " $n+1$ equally distributed points" are the images of the $(n+1)$ st roots of unity under the conformal map of $|w|>1$ onto the exterior of $C$ by the function $w=\phi(z)$. 


$$
\left|F_{m}(z)-P_{n}(z)\right| \leqq M_{4} R^{m} / d \mu^{n},
$$

$z$ on $C$,

$d$ being the minimum distance from $C$ to $\Gamma$. Let $n=q m$, where $q$ is a positive integer such that $r=\mu^{-q} R<1$; then

$$
\left|F_{m}(z)-P_{q m}(z)\right| \leqq M_{5} R^{m^{m} \mu^{-q m}}=M_{5} r^{m}, \quad z \text { on } C .
$$

Combining (8.01) and (8.03) we have

$$
\left|f(z)-P_{q m}(z)\right| \leqq M_{1} / m^{k+1}+M_{5} r^{m} \leqq M_{6} / m^{k+1}, \quad z \text { on } \bar{C},
$$

since $r<1$. Define the polynomials $\pi_{n}(z)$ in the statement of the theorem as follows:

$$
\begin{aligned}
& \pi_{n}(z) \equiv 0, \\
& n=1,2, \cdots, q-1 ; \\
& \pi_{q j+h}(z) \equiv P_{q j}(z), \\
& h=0,1, \cdots, q-1 ; j=1,2, \cdots .
\end{aligned}
$$

Since there exists a constant $M_{7}$ such that $1 / j^{k+1} \leqq M_{7} /(q j+h)^{k+1}$, the theorem follows.

For the converse problem we prove

Theorem 8.2. Let $C$ be a curve of Type $\mathrm{D}$, and let $u(z)$ be defined in $\bar{C}$. Let harmonic polynomials $p_{n}(z)$ exist such that

$$
\left|u(z)-p_{n}(z)\right| \leqq M / n^{k+\alpha},
$$

$z$ in $\bar{C}$.

Then $u(z) \in L(0, \alpha)$ on $C$ for $0<\alpha<1$ and $u(z) \in \log (0,1)$ on $C$ for $\alpha=1$. Set $f(z)=u(z)+i v(z)$, where $v(z)$ is conjugate to $u(z)$ in $\bar{C}$. Then for $k+\alpha \geqq 1$, $f(z) \in L_{A}(k, \alpha)$ on $\bar{C}$ if $0<\alpha<1$ and $f(z) \in \log _{A}(k, 1)$ on $\bar{C}$ if $\alpha=1$.

We establish first $\left({ }^{16}\right)$

Theorem 8.3. Let $C$ be a curve of Type D. Let $u(z)$ be defined in $\bar{C}$. Let harmonic polynomials $p_{n}(z)$ exist such that

$$
\left|u(z)-p_{n}(z)\right| \leqq M / n^{\beta}, \quad \quad z \text { in } \bar{C}, \beta>1 .
$$

Set $f(z)=u(z)+i v(z)$, where $v(z)$ is conjugate to $u(z)$ in $\bar{C}$. Then $f(z)$ is analytic interior to $C$, continuous in $\bar{C} ; f^{\prime}(z)$ exists in $\bar{C}$ and satisfies the condition

$$
\left|f^{\prime}(z)-\pi_{n}^{\prime}(z)\right| \leqq M_{1} / n^{\beta-1}, \quad z \text { in } \bar{C},
$$

where $\pi_{n}(z)=p_{n}(z)+i q_{n}(z), q_{n}(z)$ conjugate to $p_{n}(z)$.

The harmonicity interior to $C$ and continuity in $\bar{C}$ of $u(z)$ and thus the analyticity of $f(z)$ interior to $C$ follow from (8.05). Let $z_{0}$ be a point interior to $C$. We suppose without loss of generality $q_{n}\left(z_{0}\right)=v\left(z_{0}\right)$. Let $\nu$ and $\mu$ be any two positive integers such that $\nu>\mu$. Use of the triangle inequality yields from $(8.05)$

(16) The proofs of Theorems 8.2 and 8.3 are similar in many aspects to those of the analogous theorems for analytic functions in, for example, Sewell $[1, \S 3.4]$. The reader is referred to the latter reference for details. 


$$
\left|p_{v}(z)-p_{\mu}(z)\right| \leqq 2 M / \mu^{\beta}, \quad z \text { in } \bar{C} .
$$

Hence by Theorem 7.5 we have

$$
\left|\pi_{\nu}(z)-\pi_{\mu}(z)\right| \leqq L(\log \nu) / \mu^{\beta}, \quad \quad z \text { in } \bar{C},
$$

where $L$ is independent of $\nu$ and $\mu$.

Let $n$ be arbitrary but fixed and choose an integer $m$ such that $2^{m-1}$ $\leqq n<2^{m}$. By virtue of inequality (8.05) we may write

(8.09) $f(z)-\pi_{n}(z)=\left[\pi_{2^{m}}(z)-\pi_{n}(z)\right]+\left[\pi_{2^{m+1}}(z)-\pi_{2^{m}}(z)\right]+\cdots$,

for it follows from the Weierstrass $M$-test that the series converges uniformly in $\bar{C}$, since using (8.08) we have

$$
\begin{aligned}
& \left|\pi_{2^{m}(z)}-\pi_{n}(z)\right|+\left|\pi_{2^{m+1}(z)}-\pi_{2^{m}}(z)\right|+\cdots \\
& \leqq L \log 2\left[\frac{m}{n^{\beta}}+\frac{m+1}{2^{m \beta}}+\frac{m+2}{2^{(m+1) \beta}}+\cdots\right] \leqq L_{1} m / 2^{m \beta}, \quad z \text { in } \bar{C} .
\end{aligned}
$$

The continuity of $f(z)$ in $\bar{C}$ is now evident. Note that so far we have used merely the hypothesis $\beta>0$ rather than the stronger hypothesis $\beta>1$ of Theorem 8.3. In the remainder of the proof of Theorem 8.3, however, the requirement $\beta>1$ is essential.

From Theorem 7.2 and inequality (8.07) it follows that

$$
\left|\pi_{\nu}^{\prime}(z)-\pi_{\mu}^{\prime}(z)\right| \leqq L_{2} \nu / \mu^{\beta},
$$

$z$ in $\bar{C}$.

A similar procedure to the one just used shows that the series of derivatives converges uniformly in $\bar{C}$ :

$$
g(z)=\pi_{n}^{\prime}(z)+\left[\pi_{2^{m}}^{\prime}(z)-\pi_{n}^{\prime}(z)\right]+\left[\pi_{2^{m+1}}^{\prime}(z)-\pi_{2^{m}}^{\prime}(z)\right]+\cdots,
$$

and we have

$$
\begin{aligned}
\left|g(z)-\pi_{n}^{\prime}(z)\right| & \leqq L_{2}\left[\frac{2^{m}}{n^{\beta}}+\frac{2^{m+1}}{2^{m \beta}}+\frac{2^{m+2}}{2^{(m+1) \beta}}+\cdots\right] \\
& \leqq L_{3} /\left(2^{m}\right)^{(\beta-1)} \leqq L_{3} / n^{\beta-1}, \quad z \text { in } \bar{C} .
\end{aligned}
$$

Let $\zeta$ be a fixed point on $C$. Since the series of derivatives converges uniformly on $C$, we can integrate term-by-term along $C$ between the limits $\zeta$ and $z$ on $C$. Thus

$$
\begin{aligned}
& \int_{\zeta}^{z} g(z) d z=\left[\pi_{n}(z)-\pi_{n}(\zeta)\right] \\
& +\left[\pi_{2^{m}}(z)-\pi_{n}(z)-\left(\pi_{2^{m}}(\zeta)-\pi_{n}(\zeta)\right)\right]+\cdots \\
& =f(z)-f(\zeta) \text {. }
\end{aligned}
$$


Since the one-dimensional derivative on $C$ of the left-hand member of (8.12) exists and equals $g(z)$ (cf. the proof of Theorem 2.1), the equation $f^{\prime}(z)=g(z)$ follows on $C$. Applying Theorem 2.2 we have for the two-dimensional derivative $f^{\prime}(z)=g(z)$ in $\bar{C}$. Inequality (8.06) follows from (8.11), so Theorem 8.3 is established.

To prove Theorem 8.2 we consider first the case $k=0,0<\alpha<1$. We write from (8.04)

$$
u(z)=p_{2}(z)+\left(\sum_{m=1}^{\mu}+\sum_{m=\mu+1}^{\infty}\right)\left[p_{2^{m+1}}(z)-p_{2^{m}}(z)\right], \quad z \text { on } \bar{C},
$$

where $\mu$ is a positive integer to be determined later.

We have $\left|p_{2}(z)\right| \leqq L_{4}, z$ in $\bar{C}$; hence by Theorem 7.4

$$
\left|\pi_{2}\left(z_{1}\right)-\pi_{2}\left(z_{2}\right)\right| \leqq L_{5}\left|z_{1}-z_{2}\right|, \quad z_{1}, z_{2} \text { on } \bar{C},
$$

where $L_{5}$ is independent not only of $n, z_{1}$, and $z_{2}$, but also of $\mu$.

From (8.07) and Theorem 7.4 we obtain in the notation of Theorem 7.4

$$
\begin{aligned}
& \sum_{m=1}^{\mu}\left|\left[p_{2^{m+1}}\left(z_{1}\right)-p_{2^{m}}\left(z_{1}\right)\right]-\left[p_{2^{m+1}}\left(z_{2}\right)-p_{2^{m}}\left(z_{2}\right)\right]\right| \\
& \quad \leqq\left(4 M A / \delta_{0}\right)\left|z_{1}-z_{2}\right| \sum_{m=1}^{\mu} 2^{m(1-\alpha)} \leqq L_{6} 2^{\mu(1-\alpha)}\left|z_{1}-z_{2}\right|, \quad z_{1}, z_{2} \text { on } \bar{C},
\end{aligned}
$$

where $L_{6}$ is independent of $\mu$. Inequality (8.07) yields for $z_{1}, z_{2}$ on $\bar{C}$,

$$
\begin{aligned}
\sum_{m=\mu+1}^{\infty}\left|p_{2^{m+1}}\left(z_{1}\right)-p_{2^{m}}\left(z_{1}\right)\right|+\sum_{m=\mu+1}^{\infty} \mid p_{2^{m+1}}\left(z_{2}\right)- & p_{2^{m}}\left(z_{2}\right) \mid \\
& \leqq 4 M \sum_{m=\mu+1}^{\infty} 2^{-m \alpha}<L_{7} / 2^{\mu \alpha}
\end{aligned}
$$

$L_{7}$ independent of $\mu$. Hence

(8.13) $\left|u\left(z_{1}\right)-u\left(z_{2}\right)\right| \leqq L_{5}\left|z_{1}-z_{2}\right|+L_{6} 2^{\mu(1-\alpha)}\left|z_{1}-z_{2}\right|+L_{7} / 2^{\mu \alpha}$,

$z_{1}, z_{2}$ on $\bar{C}$.

If $k=0, \alpha=1$, we use for $f(z)$ the expansion (8.09) with $n=2$. We have as a consequence of (8.07) and Theorem 7.4

$$
\sum_{m=1}^{\mu}\left|\left[\pi_{2^{m+1}}\left(z_{1}\right)-\pi_{2^{m}}\left(z_{1}\right)\right]-\left[\pi_{2^{m+1}}\left(z_{2}\right)-\pi_{2^{m}}\left(z_{2}\right)\right]\right| \leqq L_{8} \mu\left|z_{1}-z_{2}\right|,
$$

$z_{1}, z_{2}$ on $\bar{C}$.

By the use of (8.10) we thus have

(8.14) $\left|f\left(z_{1}\right)-f\left(z_{2}\right)\right| \leqq L_{5}\left|z_{1}-z_{2}\right|+L_{8} \mu\left|z_{1}-z_{2}\right|+2 L_{1} \mu / 2^{\mu}, z_{1}, z_{2}$ on $\bar{C}$. 
Since $C$ is rectifiable, it is sufficient to consider $\left|z_{1}-z_{2}\right|<1 / 2$. Let $\left|z_{1}-z_{2}\right|$ $<1 / 2$ be given arbitrarily. Choose $\mu$ so that $\left|z_{1}-z_{2}\right|<1 / 2^{\mu} \leqq 2\left|z_{1}-z_{2}\right|$. Then $2^{\mu(1-\alpha)} \leqq\left|z_{1}-z_{2}\right|^{\alpha-1}$ and $1 / 2^{\mu \alpha} \leqq L_{9}\left|z_{1}-z_{2}\right|^{\alpha}$ and $\mu \log 2<|\log | z_{1}-z_{2}||$. Substituting these in (8.13) and (8.14) we obtain Theorem 8.2 for $k=0$.

Now let $k$ be positive. By iterating Theorem 8.3 we obtain from (8.04)

$$
\left|f^{(k)}(z)-\pi^{(k)}(z)\right| \leqq M_{2} / n^{\alpha},
$$

Thus

$$
\left|\pi_{2^{m+1}}^{(k)}(z)-\pi_{2^{m}}^{(k)}(z)\right| \leqq 2 M_{2} / 2^{m \alpha}, \quad z \text { in } \bar{C} .
$$

By use of inequality (8.15) and the expansion

$$
f^{(k)}(z)=\pi_{2}^{(k)}(z)+\sum_{m=1}^{\infty}\left[\pi_{2^{m+1}}^{(k)}(z)-\pi_{2^{m}}^{(k)}(z)\right]
$$

we prove in a manner analogous to that in which the theorem for the case $k=0$ was proved, that $f(z) \in L_{A}(k, \alpha)$ on $\bar{C}$ if $0<\alpha<1$ and $f(z) \in \log _{A}(k, 1)$ on $\bar{C}$ if $\alpha=1$. Under the hypothesis of Theorem 8.2 we have, clearly, from Theorems 8.2 and 2.3 that $D^{k} u(z), k \geqq 1$, satisfies the condition

$$
\left|D^{k} u\left(z_{1}\right)-D^{k} u\left(z_{2}\right)\right| \leqq L_{1}\left|z_{1}-z_{2}\right|^{\alpha}|\log | z_{1}-z_{2}||^{\beta}, \quad z_{1}, z_{2} \text { on } \bar{C},
$$

where $\beta=1$ if $\alpha=1$ and $\beta=0$ if $0<\alpha<1$.

The case $k=0,0<\alpha<1$, which is not fully treated in Theorem 8.2, can be satisfactorily handled under a slightly stronger hypothesis on $C$. We denote as usual by $z=\chi(w)$ the function which maps $|w|<1$ one-to-one and conformally onto the interior of $C$.

THEOREM 8.4. Let the hypothesis of Theorem 8.2 be satisfied where we now assume $0<k+\alpha<1$. Suppose there exist positive constants $L_{1}$ and $L_{2}$ such that

$$
L_{1}\left|w_{1}-w_{2}\right| \leqq\left|\chi\left(w_{1}\right)-\chi\left(w_{2}\right)\right| \leqq L_{2}\left|w_{1}-w_{2}\right|,\left|w_{1}\right|=\left|w_{2}\right|=1 .
$$

Then $f(z) \in L_{A}(0, \alpha)$ on $\bar{C}$.

Theorem 8.2 and (8.16) yield

$$
\left|u\left(z_{1}\right)-u\left(z_{2}\right)\right| \leqq M\left|z_{1}-z_{2}\right|^{\alpha} \leqq M_{1}\left|w_{1}-w_{2}\right|^{\alpha}, \quad z_{1}, z_{2} \text { on } C,
$$

where $z_{j}=\chi\left(w_{j}\right)$. Hence $u[\chi(w)] \in L(0, \alpha)$ on $|w|=1$, and therefore by Theorem 3.1, $f[\chi(w)] \in L_{A}(0, \alpha)$ on $|w|=1$. Thus by the use of Theorem 2.2 and inequality (8.16) we conclude

$$
\left|f\left(z_{1}\right)-f\left(z_{2}\right)\right| \leqq M_{2}\left|w_{1}-w_{2}\right|^{\alpha} \leqq M_{3}\left|z_{1}-z_{2}\right|^{\alpha}, \quad z_{1}, z_{2} \text { on } \bar{C} .
$$

Under a stronger hypothesis on $C$, Theorems 8.2 and 2.4 yield

THEOREM 8.5. Let $C$ be a curve of Type $\mathrm{D}$ whose equation can be written in the form $z=t(s)$, where $t^{(k)}(s)$ exists and satisfies a Lipschitz condition of order 
$\alpha$. Let $u(z)$ be defined on $\bar{C}$. Let harmonic polynomials $p_{n}(z)$ exist such that

$$
\left|u(z)-p_{n}(z)\right| \leqq M / n^{k+\alpha},
$$

$z$ on $\bar{C}$.

If $0<\alpha<1$, then $u(z) \in L(k, \alpha)$ on $C$; if $\alpha=1$, then $u(z) \in \log (k, 1)$ on $C$.

To obtain Theorem 8.5 for $0<\alpha<1, k \geqq 1$, it is necessary to make the hypothesis on $C$ that $t^{(k)}(s)$ exists and satisfies a Lipschitz condition of order $\alpha$. For if $t^{(k)}(s)$ does not exist and satisfy such a condition, either $\Re\left[t^{(k)}(s)\right]$ or $\Im\left[t^{(k)}(s)\right]$ fails to exist and satisfy a Lipschitz condition of order $\alpha$. We assume for definiteness the former, and choose $u(z) \equiv x, p_{n}(z) \equiv x, n=1,2, \cdots$; hence $u(z)$ satisfies (8.17). We have $u(z)=\Re[t(s)]$ on $C$, so $u(z) \notin L(k, \alpha)$ on $C$.

Theorem 8.5 can be established for the case $\alpha=1$ if $t^{(k)}(s)$ is required merely to satisfy a condition of the form (2.09) with $\alpha=\beta=1$. A similar remark applies in succeeding corresponding situations.

The method of splitting the series at $\mu$ was employed by de la Vallée Poussin [1] in studying real periodic functions of period $2 \pi$. The method of proof of Theorem 8.3 was used by Montel [1].

Theorems $8.2,8.3,8.4,8.5$ are proved for a single closed region bounded by a curve $C$ possessing certain continuity properties, but the same theorems clearly hold for a finite number of such regions which are mutually exterior.

9. Problem $\beta$, preliminaries. We state for reference the following two theorems due respectively to Walsh [4, p. 92] and M. Riesz [1, p. 220]:

Theorem 9.1. Let $C$ be a rectifiable Jordan curve and let $\pi_{n}(z)$ be a polynomial in $z$ of degree $n$ such that

$$
\int_{C}\left|\pi_{n}(z)\right|^{p}|d z| \leqq K^{p}, \quad p>0 .
$$

Then $\left|\pi_{n}(z)\right| \leqq L K R^{n}, z$ on $C_{R}, R>1$, where $L$ is a constant which depends on $C$, $R$, and $p$ but is independent of $\pi_{n}(z), n$, and $z$.

THEOREM 9.2. Let $f(z)=u(z)+i v(z), v(0)=0$, be analytic in $|z|<1$ and continuous in $|z| \leqq 1$. Then if $p>1$ we have

$$
\int_{0}^{2 \pi}\left|v\left(e^{i \theta}\right)\right| p d \theta \leqq M_{p} \int_{0}^{2 \pi}\left|u\left(e^{i \theta}\right)\right|^{p} d \theta
$$

where $M_{p}$ depends only on $p\left({ }^{17}\right)$.

We shall need the following general result on the derivative of a mapping function:

THEOREM 9.3. Let $C$ be a rectifiable Jordan curve. Let $w=\Omega(z)$ map the interior of $C$ one-to-one and conformally onto $|w|<1$. Then for almost every

(17) Theorem 9.2 is clearly valid for an arbitrary circle where $M_{p}$ is independent of the radius. 
point $z_{1}$ of $C$ both the one-dimensional derivative of $\Omega(z)$ on $C$ and the two-dimensional derivative of $\Omega(z)$ exist and have as common value the limit of $\Omega^{\prime}(z)$ as $z$ interior to $C$ approaches $z_{1}$ in angle.

Let $z=\chi(w)$ denote the function inverse to $w=r e^{i \theta}=\Omega(z)$. It is well known [Privaloff 2, pp. 84-85] that $\chi(w)$ is an absolutely continuous function of $\theta$ on $|w|=1$, and hence that $\chi^{\prime}(w)$ exists in the one-dimensional sense almost everywhere on $|w|=1$. Privaloff $[2$, p. 81] proves that for almost every point $w_{1}$ on $|w|=1$ we have $\lim \chi^{\prime}(w)=\chi^{\prime}\left(w_{1}\right)$ where $w$ in $|w|<1$ approaches $w_{1}$ in angle. Moreover [Lusin and Privaloff 1, p. 164] we have $\chi^{\prime}(w) \neq 0$ almost everywhere on $|w|=1$. We define measure on $C$ in terms of arc-length $s$. At almost every point of $C$ the transformation $w=\Omega(z)$ is conformal [Privaloff 2, p. 85]. It follows that at almost every point $z_{1}$ on $C$ the one-dimensional derivative $\Omega^{\prime}\left(z_{1}\right)$ on $C$ exists and equals $1 / \chi^{\prime}\left[\Omega\left(z_{1}\right)\right]$, and $\lim \Omega^{\prime}(z)=\Omega^{\prime}\left(z_{1}\right)$ where $z$ interior to $C$ approaches $z_{1}$ in angle.

To establish Theorem 9.3 it is thus sufficient to prove

$$
\lim _{z \rightarrow z_{1}, z \text { on } \bar{C}} \frac{\Omega(z)-\Omega\left(z_{1}\right)}{z-z_{1}}=\Omega^{\prime}\left(z_{1}\right),
$$

where $z_{1}$ is an arbitrary point of $C$ at which the one-dimensional derivative $\Omega^{\prime}\left(z_{1}\right)$ exists. It follows from the existence of $\Omega^{\prime}\left(z_{1}\right)$ that there exists an $\eta>0$ such that for $z$ on $C$ and $0<\left|z-z_{1}\right| \leqq \eta$ we have

$$
\left|\frac{\Omega(z)-\Omega\left(z_{1}\right)}{z-z_{1}}\right| \leqq\left|\Omega^{\prime}\left(z_{1}\right)\right|+1 .
$$

Let $\gamma_{1}$ denote the closed arc of $C$ which contains $z_{1}$ and whose end-points are the two first points of intersection of $C$ and the circle $\left|z-z_{1}\right|=\eta$ as one proceeds along $C$ from $z_{1}$ in the positive and in the negative directions. Let $\gamma_{2}$ be a Jordan arc whose interior points lie interior to $C$ and whose end points are the end points of $\gamma_{1}$; we denote by $d(>0)$ the minimum distance from $z_{1}$ to $\gamma_{2}$.

On $\gamma_{2}$ we have

$$
\left|\frac{\Omega(z)-\Omega\left(z_{1}\right)}{z-z_{1}}\right| \leqq \frac{2}{d} .
$$

By (9.02) and (9.03) we thus have [Warschawski 1] for $z$ in the closed interior of $\gamma_{1}+\gamma_{2}, z \neq z_{1}$,

$$
\left|\frac{\Omega(z)-\Omega\left(z_{1}\right)}{z-z_{1}}\right| \leqq M_{1} .
$$

Equality (9.01) now follows from a result due to Lindelöf [1].

THEOREM 9.4. Let $C$ be a rectifiable Jordan curve, and let $z_{0}$ be a point interior 
to $C$. Let $g\left(z_{0}, z\right)$ denote Green's function for the interior of $C$ with pole at $z_{0}$, and let $\nu$ denote the inner normal. In the notation of Theorem 9.3 we suppose $\Omega\left(z_{0}\right)=0$. Then almost everywhere on $C$ we have $\partial g\left(z_{0}, z\right) / \partial \nu \equiv\left|\Omega^{\prime}(z)\right|$.

The function $g\left(z_{0}, z\right)$ is chosen to become positively infinite at $z_{0}$.

Let $z_{1}$ be a point of $C$ at which $\Omega^{\prime}\left(z_{1}\right)(\neq 0)$ exists in the one-dimensional and hence in the two-dimensional sense; by Theorem 9.3 this condition is satisfied almost everywhere on $C$. Then $C$ has a tangent at $z_{1}$. We compute $\partial g / \partial \nu$, where $\nu$ is length measured along a line segment $\Gamma$ in $\bar{C}$ normal to $C$ at $z_{1}$. The image $\Gamma^{\prime}$ in the $w$-plane of $\Gamma$ is an arc normal to the circle $|w|=1$; from the continuity of $\Omega^{\prime}(z)$ on $\Gamma$ (Theorem 9.3) it follows that $\Gamma^{\prime}$ has a continuously turning tangent, and hence that arc-length $\sigma$ and chord on $\Gamma^{\prime}$ are equivalent infinitesimals. Thus we have $\left(w=r e^{i \theta}\right)$

$$
\left.\frac{\partial g}{\partial \nu}\right|_{z=z_{1}}=\frac{\partial g}{\partial \sigma} \cdot \frac{d \sigma}{|d w|}\left|\frac{d w}{d z}\right|=\frac{\partial \log r}{\partial r}\left|\Omega^{\prime}\left(z_{1}\right)\right|=\left|\Omega^{\prime}\left(z_{1}\right)\right| ;
$$

here $\partial g / \partial \sigma$ indicates the derivative along $\Gamma^{\prime}$; in the $w$-plane this derivative at $w=\Omega\left(z_{1}\right)$ on $|w|=1$ for the function $g\left(z_{0}, z\right) \equiv-\log r$ is equal to the partial derivative $\partial \log r / \partial r=1$.

The value at $z_{0}$ of an arbitrary function $u(z)$ harmonic interior to $C$ and continuous on $\bar{C}$ can be expressed as

$$
u\left(z_{0}\right)=\frac{1}{2 \pi} \int_{0}^{2 \pi} u[\chi(w)] d \theta .
$$

Since $C$ is rectifiable, $\theta=\tau(s)$ is absolutely continuous and monotonically increasing [Privaloff 2, p. 85]. Thus we have

$$
u\left(z_{0}\right)=\frac{1}{2 \pi} \int_{C} u(z) \tau^{\prime}(s) d s ;
$$

here and in the remainder of this section all integrals are Lebesgue integrals.

On $|w|=1$ we have $w=e^{i \tau(s)}$. Almost everywhere on $C$ the one-dimensional derivative $\Omega^{\prime}(z)$ exists, and [Lebesgue] $|d s / d z|=1$. There follows almost everywhere on $C$

$$
\left|\Omega^{\prime}(z)\right|=\left|\frac{d w}{d z}\right|=\left|\frac{d w}{d s} \frac{d s}{d z}\right|=\tau^{\prime}(s) .
$$

Thus it follows (Theorem 9.4) that

$$
\begin{aligned}
u\left(z_{0}\right) & =\frac{1}{2 \pi} \int_{C} u(z)\left|\Omega^{\prime}(z)\right| d s \\
& =\frac{1}{2 \pi} \int_{C} u(z) \frac{\partial g\left(z_{0}, z\right)}{\partial \nu} d s
\end{aligned}
$$


this first equation is a transform of Gauss's mean value theorem and is mentioned by Smirnoff [1]; the second equation expresses a very general form of Green's theorem involving $\partial g / \partial \nu$. A proof of this second equation recently given by $T$ suji [1] seems inadequate.

We are now in a position to extend M. Riesz's theorem to an arbitrary rectifiable Jordan curve:

THEOREM 9.5. Under the conditions of Theorem 9.4, let $f(z)=u(z)+i v(z)$, $v\left(z_{0}\right)=0$, be analytic interior to $C$ and continuous on $\bar{C}$. Then we have for $p>1$

$$
\begin{aligned}
& \int_{C}|v(z)|^{p} \frac{\partial g\left(z_{0}, z\right)}{\partial \nu} d s \leqq M_{1} \int_{C}|u(z)|^{p} \frac{\partial g\left(z_{0}, z\right)}{\partial \nu} d s, \\
& \int_{C}|f(z)|^{p} \frac{\partial g\left(z_{0}, z\right)}{\partial \nu} d s \leqq M_{2} \int_{C}|u(z)|^{p} \frac{\partial g\left(z_{0}, z\right)}{\partial \nu} d s,
\end{aligned}
$$

where $M_{1}$ and $M_{2}$ depend only on $p$.

Inequality (9.04) is a consequence of Theorems 9.2, 9.4, and the properties of $\tau(s)$ already discussed. Inequality (9.05) is a consequence of (9.04) and the well known inequality

$$
|\zeta+\eta|^{p} \leqq 2^{p-1}|\zeta|^{p}+2^{p-1}|\eta|^{p}
$$

Definition 9.1. A curve $C$ is said to be of Type B if $C$ is a rectifiable Jordan curve such that for some $q>0$ the integral $\int_{C}\left[\partial g\left(z_{0}, z\right) / \partial \nu\right]^{-q} d s$ exists.

This condition is in fact not dependent on the choice of $z_{0}$, as follows from Theorem 9.4 and consideration of an arbitrary map of the closed region $|w|$ $\leqq 1$ onto itself.

TheOREM 9.6. Let the curve $C$ be of Type B. Let $z_{0}$ be a point interior to $C$ and let $\pi_{n}(z)=p_{n}(z)+i q_{n}(z), q_{n}\left(z_{0}\right)=0$, be an arbitrary polynomial in $z$ of degree $n$. If $\left|p_{n}(z)\right| \leqq K, z$ on $C$, then

$$
\left|\pi_{n}(z)\right| \leqq M K R^{n}, \quad \quad z \text { on } \bar{C}_{R}, R>1 .
$$

We choose $q$ as in Definition 9.1. The use of Hölder's inequality and Theorem 9.5 yields

$$
\begin{aligned}
\int_{C}\left|\pi_{n}(z)\right| d s \leqq & {\left[\int_{C}\left|\pi_{n}(z)\right| 1+1 / q \frac{\partial g\left(z_{0}, z\right)}{\partial \nu} d s\right]^{q /(q+1)} } \\
& \cdot\left[\int_{C}\left[\frac{\partial g\left(z_{0}, z\right)}{\partial \nu}\right]^{-q} d s\right]^{1 /(q+1)} \\
\leqq & M_{1}\left[\int_{C}\left|p_{n}(z)\right|^{1+1 / q} \frac{\partial g\left(z_{0}, z\right)}{\partial \nu} d s\right]^{q /(q+1)} \leqq M_{9} K .
\end{aligned}
$$

Theorem 9.6 now follows from Theorem 9.1. 
10. Problem $\beta$. We are now ready to study Problem $\beta$ for sets more general than circles. Let $C$ consist of a finite number of mutually exterior Jordan curves. As in $\S 8$ let the function $w=\phi(z)$, with inverse $z=\psi(w)$, map the complement of $\bar{C}$ conformally but not necessarily uniformly onto $|w|>1$ so that the points at infinity in the two planes correspond to each other. Denote by $C_{R}$ the locus $|\phi(z)|=R>1$ lying exterior to $C$. We shall use the following definition due to Walsh and Sewell [1]:

Definition 10.1. The set $C$ is called a contour $\left({ }^{18}\right)$ if $C$ consists of the mutually exterior Jordan curves $C_{1}, C_{2}, \cdots, C_{\lambda}$ having the properties

(i) each $C_{j}$ has a tangent at every point;

(ii) the function

$$
\log \left|\left[\psi(w)-\psi\left(w^{\prime}\right)\right] /\left(w-w^{\prime}\right)\right|
$$

for each possible definition of $\psi(w)$ and $\psi\left(w^{\prime}\right)$ is locally bounded in the twodimensional sense for $|w|=1$ and $\left|w^{\prime}\right| \geqq 1$ in the neighborhood of each point of continuity of $\psi(w)$;

(iii) the function (10.01) satisfies on each arc $\gamma_{j}$ of $\gamma:|w|=1$ corresponding to a curve $C_{j}$ of $C$, a Lipschitz condition of order unity in $w$, uniformly with respect to $w^{\prime}$ on any closed arc of $\left|w^{\prime}\right|=1$ interior to a $\gamma_{k}$.

Theorem 10.1. Let $C$ and $C_{R}, R>1$, be contours. Let $u(z) \in L(k, \alpha), 0<\alpha$ $\leqq 1$, on $C_{R}$. Set $f(z)=u(z)+i v(z)$, where $v(z)$ is conjugate to $u(z)$ in $\bar{C}_{R}$. Then there exist polynomials $\pi_{n}(z)$ in $z$ such that

$$
\left|f(z)-\pi_{n}(z)\right| \leqq L / n^{k+\alpha} R^{n}
$$

z on $\bar{C}$.

By Theorem 8.1 there exist polynomials $P_{n}(z)$ in $z$ such that

$$
\left|f(z)-P_{n}(z)\right| \leqq M / n^{k+\alpha}, \quad z \text { on } \bar{C}_{R} \text {. }
$$

Denote by $\pi_{n}(z)$ the polynomial in $z$ of degree $n$ which interpolates to $f(z)$ in $n+1$ points chosen equally distributed on $C$. Then in the notation of $\S 8$ we have [Walsh and Sewell 1, Theorem 3.1]

$$
f(z)-\pi_{n}(z)=\frac{1}{2 \pi i} \int_{C_{R}} \frac{\omega_{n}(z)\left[f(t)-P_{n}(t)\right]}{\omega_{n}(t)(t-z)} d t, \quad z \text { on } C .
$$

By virtue of the inequality $\left|\omega_{n}(z) / \omega_{n}(t)\right| \leqq M_{1} / R^{n}$ for $z$ on $C$ and $t$ on $C_{R}$, we thus obtain (10.02).

In the case that $C_{R}$ is a single Jordan curve, under the hypothesis of Theorem 10.1 we can, by using the above method and previously known results, actually draw more explicit conclusions. We see from the proof of Theorem 10.1 that $\pi_{n}(z)$ in (10.02) may be chosen to interpolate to $f(z)$ in $n+1$ points equidistributed on $C$. By this same general method with a suit-

${ }^{(18)}$ For a discussion of the properties of a contour and details not given in this section, we refer to Sewell [1]. 
able definition of $\omega_{n}(z)$, if $u(z) \in L(k, \alpha)$ on $C_{R}, C$ a single Jordan curve which is such that the mapping function $z=\psi(w)$ has a nonvanishing first derivative and a continuous second derivative on $|w|=1$, and if $F_{n}(z)$ is the unique polynomial of degree $n$ which interpolates to $f(z)$ in the $n+1$ roots of the Faber [1] polynomial of degree $n+1$ belonging to $C$, it follows that inequality (10.02) is valid for $\pi_{n}(z)=F_{n}(z)$; when $C$ is an analytic Jordan curve we have analogous results for the polynomial interpolating to $f(z)$ in the roots of the Tchebycheff polynomial of degree $n+1$ belonging to $C$; see Walsh and Sewell [1] for the necessary inequalities on $\left|\omega_{n}(z) / \omega_{n}(t)\right|$ in each case.

Consider next the case of approximation on the segment $-1 \leqq z \leqq+1$. As above we have

THEOREM 10.2. Let $u(z) \in L(k, \alpha), 0<\alpha \leqq 1$, on the ellipse $C_{R}$ whose foci are $(-1,0)$ and $(+1,0)$ and whose semi-sum of axes is $R>1$. Set $f(z)$ $=u(z)+i v(z), v(z)$ conjugate to $u(z)$ in $\bar{C}_{R} ;$ if $P_{n}(z)$ is the unique polynomial of degree $n$ which interpolates to $f(z)$ in the $n+1$ roots of the polynomial $\left({ }^{19}\right)$ $\cos \left[(n+1) \cos ^{-1} z\right]$, then

$$
\left|f(z)-P_{n}(z)\right| \leqq M / n^{k+\alpha} R^{n}, \quad-1 \leqq z \leqq 1 .
$$

For the converse problem we have

TheOREm 10.3. Let $C$ be a curve of Type B; suppose that $u(z)$ is defined on $\bar{C}$. Let harmonic polynomials $p_{n}(z)$ exist such that

$$
\left|u(z)-p_{n}(z)\right| \leqq M / n^{k+\alpha+1} R^{n}, \quad z \text { on } \bar{C}, R>1 \text {. }
$$

Set $f(z)=u(z)+i v(z)$, where $v(z)$ is conjugate to $u(z)$. Then $f(z) \in L_{A}(k, \alpha)$ on $\bar{C}_{R}$ if $0<\alpha<1$ and $f(z) \in \log _{A}(k, 1)$ on $\bar{C}_{R}$ if $\alpha=1$. Hence $u(z) \in L(k, \alpha)$ on $C_{R}$ if $0<\alpha<1$ and $u(z) \in \log (k, 1)$ on $C_{R}$ if $\alpha=1$.

Let $z_{0}$ be a point interior to $C$. Set $\pi_{n}(z)=p_{n}(z)+i q_{n}(z)$, where $q_{n}(z)$ is conjugate to $p_{n}(z), q_{n}\left(z_{0}\right)=v\left(z_{0}\right)$. We write

$$
f(z)=\pi_{1}(z)+\left[\pi_{2}(z)-\pi_{1}(z)\right]+\left[\pi_{3}(z)-\pi_{2}(z)\right]+\cdots .
$$

Inequality (10.03) yields

$$
\left|p_{n+1}(z)-p_{n}(z)\right| \leqq 2 M / n^{k+\alpha+1} R^{n}
$$

$z$ on $\bar{C}$

Theorem 9.6 now implies

$$
\left|\pi_{n+1}(z)-\pi_{n}(z)\right| \leqq M_{1} / n^{k+\alpha+1},
$$

we consequently have an inequality of independent interest:

$$
\left|f(z)-\pi_{n}(z)\right| \leqq M_{1} \sum_{\nu=n}^{\infty} \nu^{-(k+\alpha+1)} \leqq M_{2} / n^{k+\alpha}, \quad z \text { on } \bar{C}_{R} .
$$

(19) The polynomial $\left(1 / 2^{n-1}\right) \cos \left[n \cos ^{-1} z\right]$ is the Tchebycheff polynomial of degree $n$ belonging to the segment $(-1,+1)$ and to all the ellipses $C_{R}$; see, for example, Faber [1], Polya and Szegö [1, vol. II, pp. 75,266$]$. 
Thus by Theorems 8.2 and $8.4, f(z) \in L_{A}(k, \alpha)$ on $\bar{C}_{R}$ if $0<\alpha<1$ and $f(z)$ $\in \log _{A}(k, 1)$ on $\bar{C}_{R}$ if $\alpha=1$. The last part of the theorem follows from Theorem 2.4.

By the use of Theorem 8.3 we have from (10.04)

TheOREM 10.4. Let $C$ be a curve of Type B. Let $u(z)$ be defined on $\bar{C}$. Let harmonic polynomials $p_{n}(z)$ exist such that

$$
\left|u(z)-p_{n}(z)\right| \leqq M / n^{\beta} R^{n}, \quad \quad z \text { in } \bar{C}, \beta>2 .
$$

Set $f(z)=u(z)+i v(z)$, where $v(z)$ is conjugate to $u(z)$; set $\pi_{n}(z)=p_{n}(z)+i q_{n}(z)$, where $q_{n}(z)$ is conjugate to $p_{n}(z)$. Then $f^{\prime}(z)$ exists in $\bar{C}_{R}$ and satisfies the condition

$$
\left|f^{\prime}(z)-\pi_{n}^{\prime}(z)\right| \leqq M_{1} / n^{\beta-2}, \quad \quad z \text { in } \bar{C}_{R} .
$$

11. Problem $\beta$ for the segment $-1 \leqq z \leqq+1$. Let us study now the situation of Theorem 10.2 in more detail. The function $z=\left(w+w^{-1}\right) / 2$ maps the $w$-plane onto the $z=(x+i y)$-plane so that the circles $|w|=R$ and $|w|=1 / R$ go into the ellipse $C_{R}$. We prove

Theorem 11.1. Let $C_{R}$ be the ellipse in the z-plane whose foci are \pm 1 and whose semi-sum of axes is $R>1$. Let $u(z)$ be harmonic interior to $C_{R}$ and continuous in $\bar{C}_{R}$. Suppose there exists a trigonometric polynomial $T_{n}(\theta)$ such that

$$
\int_{0}^{2 \pi}\left|U\left(\operatorname{Re}^{i \theta}\right)-T_{n}(\theta)\right|^{p} d \theta \leqq \epsilon_{n}^{p}, \quad p>1,
$$

where $U(w)=u\left[\left(w+w^{-1}\right) / 2\right], w=r e^{i \theta}$. Then there exists a harmonic polynomial $p_{n}(z)$ such that

$$
\left|u(z)-p_{n}(z)\right| \leqq M \epsilon_{n} / R^{n}, \quad-1 \leqq z \leqq 1 .
$$

Clearly $U(w)$ is harmonic in $1 / R<|w|<R$, continuous in $1 / R \leqq|w| \leqq R$; the harmonic character of $U(w)$ in the points $w= \pm 1$ follows from the harmonic character of $U(w)$ in the deleted neighborhoods of those points and continuity in those points themselves. Moreover we have $U(w) \equiv U(1 / w)$.

The values of $u(z)$ on the segment $-1 \leqq z \leqq 1$, which are the only values of $u(z)$ to which the conclusion applies, do not uniquely define the function $U(w)$, and it is more convenient in the sequel to replace the given $U\left(r e^{i \theta}\right)$ by the function $U^{*}\left(r e^{i \theta}\right) \equiv\left[U\left(r e^{i \theta}\right)+U\left(r e^{-i \theta}\right)\right] / 2$. The function $U^{*}(w)$ is symmetric in the axis of reals in the $w$-plane: $U^{*}\left(r e^{i \theta}\right) \equiv U^{*}\left(r e^{-i \theta}\right)$, and $U^{*}(w)$ is likewise harmonic in $1 / R<|w|<R$, continuous in $1 / R \leqq|w| \leqq R$. We also have $U^{*}\left(e^{i \theta}\right)=\left[U\left(e^{i \theta}\right)+U\left(e^{-i \theta}\right)\right] / 2=u(\cos \theta)$. We similarly replace the trigonometric polynomial $T_{n}(\theta)$ by a symmetric polynomial $T_{n}^{*}(\theta) \equiv\left[T_{n}(\theta)\right.$ $\left.+T_{n}(-\theta)\right] / 2$, whence $T_{n}^{*}(\theta) \equiv T_{n}^{*}(-\theta)$, and from our hypothesis we have by inequality $(9.06)$ 


$$
\int_{0}^{2 \pi}\left|U^{*}\left(\operatorname{Re}^{i \theta}\right)-T_{n}^{*}(\theta)\right|^{p} d \theta \leqq L \epsilon_{n}^{p} .
$$

Consequently we may write

$$
\begin{aligned}
& U^{*}\left(r e^{i \theta}\right)=a_{0}+\sum_{j=1}^{\infty}\left(r^{j}+r^{-j}\right) a_{j} \cos j \theta, \quad 1 / R<r<R, \\
& a_{j}=\left[\pi\left(R^{j}+R^{-j}\right)\right]^{-1} \int_{0}^{2 \pi} U^{*}\left(R e^{i \psi}\right) \cos j \psi d \psi .
\end{aligned}
$$

These equations for the coefficients are derived by first integrating on $|w|=r, 1 / R<r<R$, equation (11.02) multiplied through by $\cos j \theta$, and then allowing $r$ to approach $R$.

We thus have

$$
\begin{aligned}
& U^{*}\left(e^{i \theta}\right)-P_{n}^{*}\left(e^{i \theta}\right)=\frac{2}{\pi} \int_{0}^{2 \pi}\left[U^{*}\left(R e^{i \psi}\right)\right.\left.-T_{n}^{*}(\psi)\right] \\
& \cdot\left[\sum_{j=n+1}^{\infty}\left(R^{j}+R^{-j}\right)^{-1} \cos j \theta \operatorname{ccs} j \psi\right] d \psi,
\end{aligned}
$$

where $P_{n}^{*}\left(r e^{i \theta}\right)$ is the sum of the first $n+1$ terms of the right-hand member of (11.02). From the Hölder inequality and (11.01) we obtain

$$
\left|U^{*}\left(e^{i \theta}\right)-P_{n}^{*}\left(e^{i \theta}\right)\right| \leqq M \epsilon_{n} / R^{n} \text {. }
$$

On $|w|=1$ the function $\cos j \theta$ is identical with a harmonic polynomial in $x$ and $y$ of degree $j$, for we have $x=\cos \theta$; hence the trigonometric identity $\cos j \theta=\sum_{\mu=0}^{j} c_{\mu} \cos ^{\mu} \theta, c_{\mu}$ real, expresses $\cos j \theta$ as a real polynomial in $x$, $-1 \leqq x \leqq+1$; on the $x$-axis, $x^{\mu}$ is a harmonic polynomial of degree $\mu$, the real part of the polynomial $z^{\mu}$. Thus $P_{n}^{*}\left(r e^{i \theta}\right)$ is on $|w|=1$ identical with a harmonic polynomial in $x$ and $y$ of degree $n$. This completes the proof of Theorem 11.1.

Just as Theorem 5.3 is a modification of Theorem 5.1 proved by the use of Abel's Lemma, so a modification of Theorem 11.1 can be proved by that same method.

For the converse problem we prove

TheOREM 11.2. Let $u(z)$ be defined on the interval $-1 \leqq z \leqq+1$, and let there exist [harmonic] polynomials $p_{n}(z)$ such that we have

$$
\left|u(z)-p_{n}(z)\right| \leqq \epsilon_{n} / R^{n}, \quad R>1,-1 \leqq z \leqq 1,
$$

where $\sum \epsilon_{\nu}$ converges. Then $u(z)$ can be defined so as to be harmonic throughout the interior of the ellipse $C_{R}$ whose foci are \pm 1 and whose semi-sum of axes is $R$. Moreover, $u(z)$ is continuous in $\bar{C}_{R}$. If in particular $\epsilon_{n}=M / n^{k+\alpha+1}$, then, on $C_{R}, u(z) \in L(k, \alpha)$ if $0<\alpha<1$ and $u(z) \in \log (k, 1)$ if $\alpha=1$; if $f(z)$ 
$=u(z)+i v(z), v(z)$ conjugate to $u(z)$, then $f(z) \in L_{A}(k, \alpha)$ on $\bar{C}_{R}$ if $0<\alpha<1$ and $f(z) \in \log _{A}(k, 1)$ on $\bar{C}_{R}$ if $\alpha=1$.

The given polynomial $p_{n}(z)$ is equal on the axis of $x$ to a polynomial in $x$ of degree $n$, hence under the transformation $z=\left(w+w^{-1}\right) / 2$, with $w=r e^{i \theta}$, may be considered as a trigonometric polynomial in $\theta$ of order $n$ involving merely terms $\cos \nu \theta, \nu=0,1, \cdots, n$. We set formally

$$
\begin{gathered}
u(z)=a_{0}+\sum_{\nu=1}^{\infty}\left(r^{\nu}+r^{-\nu}\right) a_{\nu} \cos \nu \theta \\
a_{\nu}=\frac{1}{2 \pi} \int_{0}^{2 \pi} u(\cos \theta) \cos \nu \theta d \theta
\end{gathered}
$$

and from (11.03) we deduce by the method used in proving Theorem 5.6

$$
\left|a_{\nu+1}\right| \leqq M_{1} \epsilon_{\nu} / R^{\nu} \text {. }
$$

Consequently $u(z)$ as defined by (11.04) is harmonic for $1 / R<|w|<R$, continuous for $1 / R \leqq|w| \leqq R$; on the circle $|w|=R$ we have

$$
\left|u(z)-\sum_{\nu=0}^{n}\left(R^{\nu}+R^{-\nu}\right) a_{\nu} \cos \nu \theta\right| \leqq M_{1} \sum_{\nu=n}^{\infty}\left(R^{\nu+1}+R^{-(\nu+1)}\right) \epsilon_{\nu} / R^{\nu} \leqq 2 R M_{1} \sum_{\nu=n}^{\infty} \epsilon_{\nu} .
$$

On the circle $|w|=R$ we write $\left(R^{\nu}+R^{-\nu}\right) \cos \nu \theta=\Re\left[w^{\nu}+w^{-\nu}\right]$, and $\left(w^{\nu}+w^{-\nu}\right)$ is easily expressed as a polynomial in $z$ of degree $\nu$. The remainder of the theorem follows from Theorems 8.2, 8.4, and 8.5.

Inequality (11.03) can be interpreted as dealing not merely with harmonic polynomials of degree $n$ but also with polynomials in the real variable $x$ of the same degree $n$ or polynomials in the complex variable $z$ of this same degree $n$. If we adopt the last-mentioned interpretation, we are in a position to apply the results already established by Walsh and Sewell [4] from which it follows that if $\epsilon_{n}=M / n^{k+\alpha+1}$, then the limit $f_{1}(z)$ of these polynomials belongs either to the class $L_{A}(k, \alpha)$ or $L_{A}^{\prime}(k, 1)$, defined in $\$ 12$, on the ellipse $C_{R}$ according as $\alpha$ is less than or equal to one. Consequently, $u(z) \in L(k, \alpha)$ or $L^{\prime}(k, 1)$ on $C_{R}$.

As in Theorem 11.1, the harmonic function $u(z)$ is not uniquely determined for non-real $z$ by inequality (11.03) and the values of $u(z)$ on the segment $(-1,+1)$. If $u(z) \equiv u_{0}(z)$ satisfies (11.03), so also does $u(z) \equiv u_{0}(z)$ $+M y$. If $f_{1} \equiv u_{1}+i v_{1}$ is the analytic function defined above, the function $v_{1}(z)$ vanishes on the axis of reals because the polynomials in $z$ are real there; hence $v_{1}(z)$ satisfies the functional equation $v_{1}(z) \equiv-v_{1}(\bar{z})$. It follows that $u_{1}(z)$ satisfies the functional equation $u_{1}(z) \equiv u_{1}(\bar{z})$; moreover it is easily shown that $u_{1}(z)$ is the only harmonic function satisfying both this functional equation and inequality (11.03).

We have seen that in many theorems on approximation by harmonic and 
other polynomials, overconvergence (in the sense of Walsh $[4$, p. 79]) is involved. A certain sequence converges with sufficient rapidity (geometric degree of convergence) on a given set $E$ to insure convergence not merely on the given set $E$, but also on some larger set containing $E$ in its interior. Such is not the case, and cannot be the case, with harmonic polynomials given convergent merely on a set $E$ on the axis of reals. If the numbers $N_{j}>j$ are arbitrary, the sequence of harmonic polynomials $p_{j}(z)=N_{j} y$ of respective degrees $j$ converges on the real axis to the function $u(z) \equiv 0$ so that we have

$$
\left|u(z)-p_{j}(z)\right| \leqq 1 / N_{j}, \quad-1 \leqq z \leqq 1 .
$$

Yet the sequence $p_{j}(z)$ converges in no point not on the real axis.

12. Problem $\beta$ for negative $k$. In our study $(\$ \$ 4,8)$ of Problem $\alpha$ we considered functions $u(z)$ of class $L(k, \alpha)$ on $C, k \geqq 0$; we approximated $u(z)$ and $f(z)=u(z)+i v(z)$ on $C$ itself and were consequently interested only in functions continuous on $\bar{C}$. In Problem $\beta$, however, we study approximation to $u(z)$ and $f(z)$ on $C$ with relation to the behavior of $u(z)$ and $f(z)$ on or in a neighborhood of $C_{R}$ : thus it is appropriate to relinquish the requirement of continuity on $C_{R}$ and to consider a much more general category of functions introduced by Hardy and Littlewood. Henceforth $k$ is integral but need no longer be non-negative.

DEFINITION 12.1. If $u(z), z=r e^{i \theta}$, is harmonic in $|z|<1$ and if

$$
\left|u\left(r e^{i \theta}\right)\right| \leqq L(1-r)^{k+\alpha}, \quad r<1,0<\alpha \leqq 1,
$$

where $k<0$, then we say $u(z) \in L(k, \alpha)$ on $|z|=1$.

If $f(z)$ is analytic in $|z|<1$ and if

$$
\left|f\left(r e^{i \theta}\right)\right| \leqq L_{1}(1-r)^{k+\alpha}, \quad r<1,0<\alpha \leqq 1,
$$

where $k<0$, then we say $f(z) \in L_{A}(k, \alpha)$ on $|z|=1$.

The following theorem [Walsh and Sewell 4] indicates the connection among the classes $L_{A}(k, \alpha)$ where $k$ may be positive, negative, or zero:

THEOREM 12.1. If $f(z) \in L_{A}(k, \alpha)$ on $|z|=1,0<\alpha \leqq 1$, then the derivative $f^{\prime}(z)$ is of class $L_{A}(k-1, \alpha)$ on $|z|=1$, and, unless $k+\alpha=-1$, the indefinite integral of $f(z)$ is of class $L_{A}(k+1, \alpha)$ on $|z|=1$.

Theorem 12.1 is a justification for use of the phrase " $L_{A}(k, \alpha)$ on $|z|=1$ " in Definition 12.1, for a suitably chosen iterated integral of $f(z)$ is defined on $|z|=1$; an analogous remark applies to $L(k, \alpha)$ by virtue of results to be proved later.

To take care of the exceptional case in Theorem 12.1 we make

DEFinition 12.2. The function $u(z) \in L^{\prime}(k, 1), k \geqq-1$, on $|z|=1$ provided $u(z)$ is harmonic in $|z|<1$ and $\partial^{k+2} u(z) / \partial \theta^{k+2} \in L(-2,1)$ on $|z|=1$; the function $f(z) \in L_{A}^{\prime}(k, 1), k \geqq-1$, on $|z|=1$ provided $f^{(k+2)}(z) \in L_{A}(-2,1)$ on $|z|=1$. 
We extend our classification from the unit circle to an analytic Jordan curve by conformal mapping:

Definition 12.3. Let $C$ be an analytic Jordan curve in the $z$-plane. Let $z=\chi(w)$ map $|w|<1$ one-to-one and conformally onto the interior of $C$. Then $u(z)$ is said to be of class $L(k, \alpha), k<0$, or $L^{\prime}(k, 1), k \geqq-1$, on $C$ if $u[\chi(w)]$ is of class $L(k, \alpha)$ or $L^{\prime}(k, 1)$, respectively, on $|w|=1,0<\alpha \leqq 1$. The function $f(z)$ is said to be of class $L_{A}(k, \alpha), k<0$, or $L_{A}^{\prime}(k, 1), k \geqq-1$, on $C$ if $f[\chi(w)]$ is of class $L_{A}(k, \alpha)$ or $L_{A}^{\prime}(k, 1)$, respectively, on $|w|=1,0<\alpha \leqq 1$.

This definition is independent of the particular $\chi(w)$ chosen, as the reader will easily show by considering an arbitrary map of the region $|w|<1$ onto itself; for the classes $L^{\prime}(k, 1)$ compare also Theorem 12.4 below. Moreover, Theorem 12.1 carries over where we now differentiate and integrate in the plane of the curve $C$.

If $C$ consists of several mutually exterior analytic Jordan curves, we say $u(z) \in L(k, \alpha), k<0$, or $u(z) \in L^{\prime}(k, 1), k \geqq-1$, on $C$ if $u(z) \in L(k, \alpha)$ or $u(z) \in L^{\prime}(k, 1)$, respectively, on each component of $C$; a similar definition applies to $f(z)$ and the classes $L_{A}(k, \alpha), L_{A}^{\prime}(k, 1)$.

The following theorem due to Cartwright [1] will be useful:

THEOREM 12.2. Let $f(z)=u(z)+i v(z), z=r e^{i \theta}$, be analytic in $|z|<1$ and let $v(0)=0$. If $\left|u\left(r e^{i \theta}\right)\right| \leqq L(1-r)^{-\mu}$ for $r<1$, where $\mu>0$, then $\left|v\left(r e^{i \theta}\right)\right|$ $\leqq M_{\mu} L(1-r)^{-\mu}$ for $r<1$, where $M_{\mu}$ depends only on $\mu$.

To relate the classes $L(k, \alpha)$ and $L_{A}(k, \alpha), k<0$, we thus have

THEOREM 12.3. Let $C$ be an analytic Jordan curve. Let $f(z)=u(z)+i v(z)$ be analytic interior to $C$ and suppose $k+\alpha<0,0<\alpha \leqq 1$. Then a necessary and sufficient condition that $f(z) \in L_{A}(k, \alpha)$ on $C$ is that $u(z) \in L(k, \alpha)$ on $C$.

By Definition 12.3 we need consider only the case of the unit circle. The necessity of the condition is obvious. The sufficiency follows by Theorem 12.2:

$$
\begin{gathered}
\left|v\left(r e^{i \theta}\right)-v(0)\right| \leqq M_{\mu} L(1-r)^{k+\alpha}, \\
\left|v\left(r e^{i \theta}\right)\right| \leqq M_{\mu} L(1-r)^{k+\alpha}+|v(0)| \leqq M_{1}(1-r)^{k+\alpha}, \quad r<1 .
\end{gathered}
$$

Under a hypothesis on $u(z)$ analogous to that of Theorem 12.1 on $f(z)$, Theorems 12.3 and 12.1 yield an analogous conclusion concerning the derivative and indefinite integral of $u(z)+i v(z)$. The statement and proof of such a theorem are left to the reader.

THEOREM 12.4. Let $C$ be an analytic Jordan curve. Let $f(z)=u(z)+i v(z)$ be analytic interior to $C$. Then a necessary and sufficient condition that $f(z)$ $\in L_{A}^{\prime}(k, 1), k \geqq-1$, on $C$ is that $u(z) \in L^{\prime}(k, 1)$ on $C$.

By Definition 12.3 we may limit ourselves to the case of the unit circle. The necessitv follows from Definitions 12.1 and 12.2 and the equation $\partial^{k+2} f / \partial \theta^{k+2}=\sum_{j=1}^{k+2} c_{j} z^{j} f^{(j)}(z),|z|<1$, where the $c_{j}$ are constants. To estab- 
lish the sufficiency we note that, by Theorem $12.3, \partial^{k+2} f / \partial \theta^{k+2}$ satisfies the condition $\left|\partial^{k+2} f\left(r e^{i \theta}\right) / \partial \theta^{k+2}\right| \leqq L /(1-r)$; hence $\left|\partial^{j} f\left(r e^{i \theta}\right) / \partial \theta^{j}\right| \leqq L_{1} /(1-r)$ for $j=1, \cdots, k+1$. Thus from the equation $f^{(k+2)}(z)=z^{-(k+2)} \sum_{j=1}^{k+2} d_{j}\left(\partial^{j} f(z) / \partial \theta^{j}\right)$, we obtain

$$
\left|f^{(k+2)}\left(r e^{i \theta}\right)\right| \leqq M r^{-(k+2)} /(1-r), \quad 0<r<1 .
$$

For $r \leqq 1 / 2$, the principle of the maximum together with (12.01) yields

$$
\left|f^{(k+2)}(z)\right| \leqq 2^{k+3} M /(1-r) ;
$$

hence we see from (12.01) that (12.02) is valid for $|z|<1$.

Theorem 12.5. Let $C$ and $C_{R}, R>1$, be contours. Let $u(z) \in L(k, \alpha), k<0$, $0<\alpha \leqq 1$, on $C_{R}$. Set $f(z)=u(z)+i v(z)$ where $v(z)$ is conjugate to $u(z)$ interior to $C_{R}$. Then there exist polynomials $\pi_{n}(z)$ in $z$ such that

$$
\left|f(z)-\pi_{n}(z)\right| \leqq L / n^{k+\alpha} R^{n},
$$

Except in the case $k=-1, \alpha=1$, Theorem 12.5 follows immediately by Theorem 12.3 from the known corresponding theorem for analytic functions [Sewell 1, Theorem 6.1.1].

Consider the case $k=-1, \alpha=1$. By hypothesis $|u(z)| \leqq M, z$ interior to $C_{R}$. Let $C^{\prime}$ be a component curve of $C_{R}$, and let $z=\chi(w), w=r e^{i \theta}$, map $|w|<1$ one-to-one and conformally onto the interior of $C^{\prime}$. We obtain by the use of Theorem 9.2

$$
\begin{aligned}
\int_{|w|=r}|f[\chi(w)]| \mid & \chi^{\prime}(w) \mid d \theta \\
& \leqq M_{1}\left[\int_{|w|=r}|f[\chi(w)]|^{2} d \theta\right]^{1 / 2} \\
& \leqq M_{2}\left[\int_{|w|=r}|f[\chi(w)]-i v[\chi(0)]|^{2} d \theta+2 \pi|v[\chi(0)]|^{2}\right]^{1 / 2} \\
& \leqq M_{2}\left[M_{3} \int_{|w|=r}|u[\chi(w)]|^{2} d \theta+2 \pi|v[\chi(0)]|^{2}\right]^{1 / 2} \\
& \leqq M_{2}\left[2 \pi M_{3} M^{2}+2 \pi|v[\chi(0)]|^{2}\right]^{1 / 2}<M_{4}, \quad r<1,
\end{aligned}
$$

where $M_{4}$ is independent of $r$. It follows from this last inequality by well known theorems that the limit of $f(z)$ as $z$ approaches a point of $C_{R}$ in angle exists for almost all points of $C_{R}$ and that $\int_{C_{R}}|f(z)| d s$ exists. Furthermore [for example, Smirnoff 1, p. 345], the Cauchy integral formula is valid for $f(z)$, where the integral is taken along $C_{R}$.

Hence if $\pi_{n}(z)$ denotes the polynomial of degree $n$ which interpolates to $f(z)$ in $n+1$ equally distributed points on $C$ we have [Walsh and Sewell 1] in the usual notation (\$8) 


$$
\begin{array}{rr}
f(z)-\pi_{n}(z)=\frac{1}{2 \pi i} \int_{C_{R}} \frac{\omega_{n}(z) f(t)}{\omega_{n}(t)(t-z)} d t, & z \text { on } C \\
\left|f(z)-\pi_{n}(z)\right| \leqq L / R^{n}, & z \text { on } \bar{C} .
\end{array}
$$

Theorem 12.4 together with known results [Walsh and Sewell 4, Theorem 7.6] yields

THEOREM 12.6. Let $C$ be an analytic Jordan curve, and let $u(z) \in L^{\prime}(k, 1)$ on $C_{R}, k \geqq-1, R>1$. Set $f(z)=u(z)+i v(z)$, where $v(z)$ is conjugate to $u(z)$ interior to $C_{R}$. Then there exist polynomials $\pi_{n}(z)$ such that

$$
\left|f(z)-\pi_{n}(z)\right| \leqq M / n^{k+1} R^{n}, \quad \text { on } \bar{C} .
$$

In the converse direction we have

Theorem 12.7. Let $C$ be a curve of Type $D$, and let $u(z)$ be defined on $\bar{C}$. Let harmonic polynomials $p_{n}(z)$ exist such that

$$
\left|u(z)-p_{n}(z)\right| \leqq M / n^{\beta} R^{n}, \quad \text { zon } \bar{C}, R>1 .
$$

If we set $f(z)=u(z)+i v(z)$, where $v(z)$ is conjugate to $u(z)$ in $\bar{C}$, and $\pi_{n}(z)=p_{n}(z)$ $+i q_{n}(z)$, where $q_{n}(z)$ is conjugate to $p_{n}(z)$, then $f(z)$ is analytic on $\bar{C}$ and

$$
\left|f^{\prime}(z)-\pi_{n}^{\prime}(z)\right| \leqq M_{1} / n^{\beta-1} R^{n}, \quad \text { on } \bar{C} \text {. }
$$

From (12.03) we obtain

$$
\begin{aligned}
& \left|p_{n+1}(z)-p_{n}(z)\right| \leqq 2 M / n^{\beta} R^{n}, \\
& \beta \geqq 0 \text {, } \\
& \left|p_{n+1}(z)-p_{n}(z)\right| \leqq 2 M /(n+1)^{\beta} R^{n}, \\
& \beta<0 \text {. }
\end{aligned}
$$

Thus by Theorem 7.2 we have

$$
\left|\pi_{n+1}^{\prime}(z)-\pi_{n}^{\prime}(z)\right| \leqq M_{1} / n^{\beta-1} R^{n}, \quad z \text { on } \bar{C} .
$$

By the method used in proving Theorem 8.3 we obtain that the sequence $\pi_{n}(z)$ converges to $f(z)$ in $\bar{C}$. Theorem 12.7 now follows by the method of Sewell [ 1 , Theorem 6.2.8].

THEOREM 12.8. Let $C$ be an analytic Jordan curve, and let $u(z)$ be defined on $\bar{C}$. Let harmonic polynomials $p_{n}(z)$ exist such that

$$
\left|u(z)-p_{n}(z)\right| \leqq M / n^{k+\alpha+1} R^{n}, \quad z \text { on } \bar{C}, R>1 .
$$

Let $f(z)$ be an analytic function such that $\Re[f(z)]=u(z)$ interior to $C_{R}$. Then $f(z) \in L_{A}(k, \alpha)$ on $C_{R}$ if $k+\alpha+1$ is not a positive integer, and $f(z) \in L_{A}^{\prime}(k, \alpha)$ on $C_{R}$ if $k+\alpha+1$ is a positive integer.

Theorem 12.8 holds for $k$ positive, negative, or zero.

Inequality (12.04) implies by Theorem 12.7 in the notation of that theorem 


$$
\left|f^{\prime}(z)-\pi_{n}^{\prime}(z)\right| \leqq M_{1} / n^{k+\alpha} R^{n}
$$

$z$ on $\bar{C}$.

If $k+\alpha$ is not a positive integer, then [Sewell 1, Theorem 6.3.1] $f^{\prime}(z)$ $\in L_{A}(k-1, \alpha)$ on $C_{R}$; thus the conclusion follows by Theorem 12.1 as extended if we have $k+\alpha \neq 0$, and by Definition 12.3 if we have $k+\alpha=0$. If $k+\alpha$ is a positive integer, then [Sewell 1, Theorem 6.3.1] $f^{\prime}(z) \in L_{A}^{\prime}(k-1, \alpha)$ on $C_{R}$; thus by Definition $12.3 f^{(k+2)}(z) \in L_{A}(-2,1)$ and hence $f(z) \in L_{A}^{\prime}(k, 1)$ on $C_{R}$.

Theorem 12.8 can be extended to the sum $C$ of a finite number of mutually exterior analytic Jordan curves, for Theorem 12.7 may be applied to each component of $C$ to obtain (12.05); Theorem 6.3.1 of Sewell [1] extends to such a set $C$.

Results on Problem $\beta$ for negative $k$ for the line segment may be obtained by the methods of this section and the preceding one; the statement and proof of such theorems are left to the reader.

13. Problem $\gamma$. We turn now to the study of the polynomials under consideration in Problem $\beta$ in $\$ 10$. Let $C$ be a contour. Let $f(z)$ be defined in $\bar{C}$. Suppose that there exist polynomials $\pi_{n}(z)$ in $z$ such that

$$
\left|f(z)-\pi_{n}(z)\right| \leqq \epsilon_{n}, \quad z \text { in } \bar{C},
$$

where the $\epsilon_{n}$ approach zero monotonically as $n$ becomes infinite. Then we have

$$
\left|\pi_{n+1}(z)-\pi_{n}(z)\right| \leqq 2 \epsilon_{n}, \quad z \text { on } \bar{C},
$$

and therefore [Walsh 4, p. 77]

$$
\left|\pi_{n+1}(z)-\pi_{n}(z)\right| \leqq 2 \epsilon_{n} R^{n}, \quad z \text { on } \bar{C}_{R}, R>1 .
$$

If we set formally

$$
f(z)=\pi_{1}(z)+\left[\pi_{2}(z)-\pi_{1}(z)\right]+\left[\pi_{3}(z)-\pi_{2}(z)\right]+\cdots,
$$

and if $\sum \epsilon_{n} R^{n}$ converges, we may consider the second member of (13.02) to define $f(z)$ on $\bar{C}_{R}$. By (13.01) we have

$$
\left|f(z)-\pi_{n}(z)\right| \leqq 2 \sum_{j=n}^{\infty} \epsilon_{j} R^{j},
$$

This method can be applied to the various sequences of polynomials studied in $\$ 10$ whose degree of convergence on $C$ is known. We have, for example, from Theorem 10.1

Theorem 13.1. Let $C$ and $C_{R}, R>1$, be contours. Let $u(z) \in L(k, \alpha), 0<\alpha$ $\leqq 1, k \geqq 1$, on $C_{R}$. Set $f(z)=u(z)+i v(z)$, where $v(z)$ is conjugate to $u(z)$ in $\bar{C}_{R}$. Then

$$
\left|f(z)-\pi_{n}(z)\right| \leqq M / n^{k+\alpha-1}, \quad \quad z \text { in } \bar{C}_{R}
$$


where $\pi_{n}(z)$ is the (unique) polynomial of degree $n$ which interpolates to $f(z)$ in $n+1$ equally distributed points on $C$.

If we choose $\epsilon_{n}=M / n^{k+\alpha+1} R^{n}, k \geqq 0$, and $\epsilon_{n}=M_{1} / n^{k+\alpha} R^{n}$ respectively, we obtain using the methods of this section and Theorem 9.6

Theorem 13.2. Let $C$ be a curve of Type B, and let $u(z)$ be defined in $\bar{C}$. Let harmonic polynomials $p_{n}(z)$ satisfy the inequalities

$$
\left|u(z)-p_{n}(z)\right| \leqq M / n^{k+\alpha+1} R^{n}, \quad z \text { on } \bar{C}, R>1, k \geqq 0 .
$$

Set $f(z)=u(z)+i v(z)$, where $v(z)$ is conjugate to $u(z)$. Let $z_{0}$ be an interior point of $C$; set $\pi_{n}(z)=p_{n}(z)+i q_{n}(z)$, where $q_{n}(z)$ is conjugate to $p_{n}(z), q_{n}\left(z_{0}\right)=v\left(z_{0}\right)$. Choose $R_{1}, 1<R_{1} \leqq R$. Then

$$
\left|f(z)-\pi_{n}(z)\right| \leqq L M R_{1}^{n} / n^{k+\alpha} R^{n}, \quad \text { zon } \bar{C}_{R_{1}},
$$

where $L$ depends on $R_{1}$ but is independent of $u(z), n, R$, and $z$; if $1<R_{1}<R$ we have furthermore

$$
\left|f(z)-\pi_{n}(z)\right| \leqq L_{1} M R_{1}^{n} / n^{k+\alpha+1} R^{n}, \quad \text { on } \bar{C}_{R_{1}},
$$

where $L_{1}$ depends also on $R$.

14. Appraisal of results. Examples. We now exhibit examples to appraise the sharpness of our results. To see that Theorem 3.1 is in a certain sense sharp for the case $u(z) \in L(k, 1)$, we may consider the function

$$
\begin{aligned}
f_{1}(z) & =(z-1) \log (1-z) & (|z| \leqq 1, z \neq 1) \\
& =0 & (z=1) .
\end{aligned}
$$

Choose the branch of the logarithm such that $-\pi / 2 \leqq \arg (1-z)<\pi / 2$. If $f_{1}(z)=u_{1}(z)+i v_{1}(z)$, we see that $u_{1}(z) \in L(0,1)$ on $|z|=1$, but $v_{1}(z) \notin L(0,1)$ on $|z|=1$. We have rather

$$
L_{1} h|\log h| \leqq\left|v_{1}\left(e^{i h}\right)-v_{1}(1)\right| \leqq L_{2} h|\log h|, \quad 0<h \leqq \delta_{1},
$$

where $L_{1}$ and $L_{2}$ are positive constants independent of $h$. Hence there can be established on $f_{1}\left(e^{i \theta}\right)$ no stronger condition expressible as a modulus of continuity than an inequality of form (2.01) with $k=0, \beta=1$. For $k>0$ the $k$ th indefinite integral of $f_{1}(z)$ yields a similar conclusion.

To see that Theorem 3.1 is sharp for the case $u(z) \in \log (k, 1)$, consider $(k=0)$ the following function or $(k>0)$ its $k$ th indefinite integral:

$$
\begin{aligned}
f_{2}(z) & =(z-1) \log ^{2}(1-z) & (|z| \leqq 1, z \neq 1) \\
& =0 & (z=1),
\end{aligned}
$$

where $-\pi / 2 \leqq \arg (1-z)<\pi / 2$. If $f_{2}(z)=u_{2}(z)+i v_{2}(z)$, then $u_{2}(z) \in \log (0,1)$ on $|z|=1$, but $v_{2}(z) \notin \log (0,1)$ on $|z|=1$. We have instead 


$$
M_{1} h \log ^{2} h \leqq\left|v_{2}\left(e^{i h}\right)-v_{2}(1)\right| \leqq M_{2} h \log ^{2} h, \quad 0<h \leqq \delta_{2},
$$

where $M_{1}$ and $M_{2}$ are positive constants independent of $h$.

We turn now to examples for the theorems on approximation. For $0<\alpha<1$ and $C$ an analytic Jordan curve, Theorem 8.5 is an exact converse of results contained in Theorem 8.1. For $\alpha=1$, however, a corresponding exact converse is impossible, as is illustrated $(k=0)$ by the function $v_{1}(z)$ defined above. The power series expansion of $f_{1}(z)$ about the origin is

$$
f_{1}(z)=z-\sum_{\nu=2}^{\infty} z^{\nu} / \nu(\nu-1), \quad|z| \leqq 1,
$$

which converges uniformly for $|z| \leqq 1$ by the Weierstrass $M$-test. For $|z| \leqq 1$ we have

$$
\begin{aligned}
& \left|f_{1}(z)-z+\sum_{\nu=2}^{n} z^{\nu} / \nu(\nu-1)\right| \leqq \sum_{n+1}^{\infty} 1 / \nu(\nu-1)=\sum_{n+1}^{\infty}\left[(\nu-1)^{-1}-\nu^{-1}\right]=1 / n, \\
& n=2,3, \cdots ; \text { thus }\left(z=r e^{i \theta}\right) \\
& \quad\left|v_{1}(z)-r \sin \theta+\sum_{\nu=2}^{n} r^{\nu} \sin \nu \theta / \nu(\nu-1)\right| \leqq 1 / n, \quad r \leqq 1 .
\end{aligned}
$$

But $v_{1}(z)$ satisfies a condition of the form (2.01) with $k=0, \beta=1$, and no stronger condition expressible as a modulus of continuity. For $k>0$ the $k$ th indefinite integral of $f_{1}(z)$ yields a similar illustration.

The exponent of $n$ cannot be improved in Theorem 10.1. Compare the examples given by Walsh and Sewell $[1$, pp. 700-702].

To see that $\alpha=1$ is exceptional in Theorems 10.3 and 12.8 we refer to the examples in Sewell [1, p. 169].

The discrepancy of unity between the exponents which occur in Theorem 10.1 and in Theorem 10.3 is inherent in the nature of the problem; compare Sewell [1, pp. 170-171].

\section{BIBLIOGRAPHY}

T. CARLeman

1. Über die Fourierkoeffizienten einer stetigen Funktion, Acta Math. vol. 41 (1918) pp. 377384.

M. L. CARTwRight

1. On analytic functions regular in the unit circle, Quart. J. Math. Oxford Ser. vol. 4 (1933) pp. 246-257.

John CuRTiss

1. A note on the degree of polynomial approximation, Bull. Amer. Math. Soc. vol. 42 (1936)

G. FABER pp. 873-878.

1. Über Tschebyscheffsche Polynome, J. Reine Angew. Math. vol. 150 (1920) pp. 79-106. 
M. FEKETE

1. Über einen Satz des Herrn Serge Bernstein, J. Reine Angew. Math. vol. 146 (1916) pp. 88-94.

D. JACKSON

1. The theory of approximation, Amer. Math. Soc. Colloquium Publications, vol. 11, New York, 1930.

C. DE la Vallée Poussin

1. Leçons sur l'approximation des fonctions d'une variable réelle, Paris, 1919.

E. LINDELÖF

1. Sur un principe général de l'analyse, Acta Societatis Scientiarum Fennicae vol. 46 (1915) No. 4.

N. Lusin and J. Privaloff

1. Sur l'unicité et la multiplicité de fonctions analytiques, Ann. Ecole Norm. (3) vol. 42 (1925) pp. 143-191.

P. MONTEL

1. Sur les polynômes d'approximation, Bull. Soc. Math. France vol. 46 (1919) pp. 151-192.

G. Pólya AND G. Szegö

1. Aufgaben und Lehrsätze aus der Analysis, Berlin, 1925.

J. Privaloff

1. Sur les fonctions conjuguées, Bull. Soc. Math. France vol. 44 (1916) pp. 100-103.

2. Sur certaines propriétés métriques des fonctions analytiques, J. Ecole Polytech. (2) vol.

M. RIESZ 24 (1924) pp. 77-112.

1. Sur les fonctions conjuguées, Math. Zeit. vol. 27 (1928) pp. 218-244.

A. C. Schaeffer and G. Szegö

1. Inequalities for harmonic polynomials in two and three dimensions, Trans. Amer. Math. Soc. vol. 50 (1941) pp. 187-225.

W. SEIDEL

1. Über die Ränderzuordnung bei konformen Abbildungen, Math. Ann. vol. 104 (1931) pp. 182-243.

W. E. SEWELI

1. Degree of approximation by polynomials in the complex domain, Annals of Mathematics Studies, vol. 9, Princeton, 1942.

V. SmirnofF

1. Sur les formules de Cauchy et de Green et quelques problèmes qui s'y rattachent, Bull. Acad. Sci. URSS. Sér. Math. vol. 7 (1932) pp. 337-371.

G. SzEGö

1. Über trigonometrische und harmonische Polynome, Math. Ann. vol. 79 (1919) pp. 323-329.

2. Über einen Satz des Herrn Serge Bernstein, Schrif ten der Königsberger Gelehrten Gesellschaft, Naturwissenschaf tliche Klasse vol. 5 (1928) pp. 59-70.

M. TsujI

1. On the Green's function, Jap. J. Math. vol. 18 (1941-1943) pp. 379-383.

J. L. WALSH

1. On the degree of approximation to a harmonic function, Bull. Amer. Math. Soc. vol. 33 (1927) pp. 591-598.

2. Über die Entwicklung einer harmonischen Funktion nach harmonischen Polynomen, J. Reine Angew. Math. vol. 159 (1928) pp. 197-209.

3. The approximation of harmonic functions by harmonic polynomials and by harmonic rational functions, Bull. Amer. Math. Soc. vol. 35 (1929) pp. 499-544.

4. Interpolation and approximation by rational functions in the complex domain, Amer. Math. Soc. Colloquium Publications, vol. 20, New York, 1935. 
5. Maximal convergence of sequences of harmonic polynomials, Ann. of Math. vol. 38 (1937) pp. 321-354.

J. L. Walsh AND W. E. Sewell

1. Sufficient conditions for various degrees of approximation by polynomials, Duke Math. J. vol. 6 (1940) pp. 658-705.

2. A note on the relation between continuity and degree of polynomial approximation in the complex domain, Bull. Amer. Math. Soc. vol. 43 (1937) pp. 557-563.

3. Note on degree of trigonometric and polynomial approximation to an analytic function, ibid. vol. 44 (1938) pp. 865-873.

4. On the degree of polynomial approximation to analytic functions: Problem $\beta$, Trans. Amer. Math. Soc. vol. 49 (1941) pp. 229-257.

S. E. WARSCHAWSKI

1. Bemerkung zu meiner Arbeit: Über das Randverhalten der Ableitung der Abbildungsfunktion bei konformer Abbildung, Math. Zeit. vol. 38 (1934) pp. 669-683.

A. Zygmund

1. Trigonometrical series, Monografje Matematyczne, vol. V, Warsaw-Lwów, 1935.

2. Smooth functions, Duke Math. J. vol. 12 (1945) pp. 47-76.

HARVARD UNIVERSITY,

Cambridge, Mass.

Special Staff, United States Army, Washington, D. C.

HARVARD UNIVERSITY,

Cambridge, Mass. 